\title{
Nef-mediated down-regulation of CD4 and HLA class I in HIV-1 subtype $C$ infection: Association with disease progression and influence of immune pressure
}

\author{
Jaclyn K. Mann ${ }^{\mathrm{a}, \mathrm{b}, 1}$, Denis Chopera ${ }^{\mathrm{a}, \mathrm{b}, \mathrm{c}, 1}$, Saleha Omarjee ${ }^{\mathrm{a}, \mathrm{b}}$, Xiaomei T. Kuang ${ }^{\mathrm{d}}$, Anh Q. Le ${ }^{\mathrm{e}}$, \\ Gursev Anmole ${ }^{\mathrm{d}}$, Ryan Danroth ${ }^{\mathrm{e}}$, Philip Mwimanzi ${ }^{\mathrm{e}}$, Tarylee Reddy ${ }^{\mathrm{f}}$, Jonathan Carlson ${ }^{\mathrm{g}}$, \\ Mopo Radebe ${ }^{\mathrm{a}, \mathrm{b}}$, Philip J.R. Goulder ${ }^{\mathrm{h}, \mathrm{i}}$, Bruce D. Walker ${ }^{\mathrm{i}, \mathrm{j}, \mathrm{k}}$, Salim Abdool Karim ${ }^{1}$, \\ Vladimir Novitsky ${ }^{\mathrm{m}, \mathrm{n}}$, Carolyn Williamson ${ }^{\mathrm{c}}$, Mark A. Brockman ${ }^{\mathrm{d}, \mathrm{e}, \mathrm{o}}$, Zabrina L. Brumme ${ }^{\mathrm{e}, \mathrm{o}}$, \\ Thumbi Ndung'u ${ }^{\text {a,b,i,p,* }}$
}

${ }^{a}$ HIV Pathogenesis Programme, University of KwaZulu-Natal, 719 Umbilo Road, Durban 4001, South Africa

${ }^{\mathrm{b}}$ KwaZulu-Natal Research Institute for Tuberculosis and HIV, University of KwaZulu-Natal, Durban 4001, South Africa

${ }^{\mathrm{c}}$ Institute of Infectious Disease and Molecular Medicine, and the Division of Medical Virology, University of Cape Town and National Health Laboratory

Services, Cape Town 7925, South Africa

${ }^{\mathrm{d}}$ Molecular Biology and Biochemistry, Simon Fraser University, Burnaby, BC, Canada V5A 156

e Faculty of Health Sciences, Simon Fraser University, Burnaby, BC, Canada V5A 1S6

${ }^{\mathrm{f}}$ Medical Research Council, Biostatistics Unit, Durban 4001, South Africa

${ }^{g}$ Microsoft Research, Los Angeles, CA 90024, United States of America

${ }^{\mathrm{h}}$ Department of Paediatrics, University of Oxford, Oxford OX1 3SY, United Kingdom

${ }^{\mathrm{i}}$ Ragon Institute of MGH, MIT and Harvard University, Cambridge, MA 02139, USA

${ }^{j}$ Massachusetts General Hospital and Harvard University, Boston, MA 02114, USA

${ }^{\mathrm{k}}$ Howard Hughes Medical Research Institute, Chevy Chase, MD 20815, USA

${ }^{1}$ Centre for the AIDS Programme of Research in South Africa, University of KwaZulu-Natal, Durban 4001, South Africa

${ }^{m}$ Department of Immunology and Infectious Diseases, Harvard School of Public Health, Boston, MA 02115, USA

n Botswana-Harvard School of Public Health AIDS Initiative Partnership for HIV Research and Education, P/Bag BO 320, Gaborone, Botswana

${ }^{\circ}$ British Columbia Centre for Excellence in HIV/AIDS, Vancouver, BC, Canada V6Z 1 Y6

${ }^{\mathrm{P}}$ Max Planck Institute for Infection Biology, Chariteplatz, D-10117 Berlin, Germany

\section{A R T I C L E I N F O}

\section{Article history:}

Received 30 May 2014

Returned to author for revisions

8 June 2014

Accepted 11 August 2014

\section{Keywords:}

HIV-1 Nef

HIV-1 subtype C

CD4 down-regulation

HLA-I down-regulation

HIV-1 disease progression

HLA-associated polymorphisms

Immune evasion

\begin{abstract}
A B S T R A C T
Nef plays a major role in HIV-1 pathogenicity. We studied HIV-1 subtype $C$ infected individuals in acute/ early $(n=120)$ or chronic $(n=207)$ infection to investigate the relationship between Nef-mediated CD4/ HLA-I down-regulation activities and disease progression, and the influence of immune-driven sequence variation on these Nef functions. A single Nef sequence per individual was cloned into an expression plasmid, followed by transfection of a T cell line and measurement of CD4 and HLA-I expression. In early infection, a trend of higher CD4 down-regulation ability correlating with higher viral load set point was observed $(r=0.19, p=0.05)$, and higher HLA-I down-regulation activity was significantly associated with faster rate of CD4 decline $(p=0.02)$. HLA-I down-regulation function correlated inversely with the number HLA-associated polymorphisms previously associated with reversion in the absence of the selecting HLA allele $(r=-0.21, p=0.0002)$. These data support consideration of certain Nef regions in HIV-1 vaccine strategies designed to attenuate the infection course.
\end{abstract}

(c) 2014 Elsevier Inc. All rights reserved.

\footnotetext{
* Corresponding author at: HIV Pathogenesis Programme, University of KwaZuluNatal, 719 Umbilo Road, Durban 4013, South Africa. Tel.: +27 31 2604727; fax: +27312604623.

E-mail address: ndungu@ukzn.ac.za (T. Ndung'u).

${ }^{1}$ These authors contributed equally to this work.
}

\section{Introduction}

HIV-1 Nef is a small accessory protein that plays a major role in viral replication and pathogenesis (Foster et al., 2011). Nef displays multiple functions in vitro, including down-regulation of cell-surface CD4 (to enhance viral spread and possibly evade antibody-dependent cell-mediated cytotoxicity of infected cells), down-regulation of HLA 
class I (HLA-I) A and B molecules (to evade CD8 + T cell responses), activation of $\mathrm{T}$ cells and $\mathrm{CD} 4$-independent enhancement of virion infectivity (Foster and Garcia, 2008; Veillette et al., 2014). The relative importance of these Nef activities in vivo remain incompletely understood, although some studies suggest that Nef-mediated CD4 downregulation, enhancement of infectivity, and one or more unknown Nef functions represent major pathogenic contributors (Iafrate et al., 2000; Watkins et al., 2013).

Nef's influence on HIV-1 pathogenesis is clearly demonstrated by an attenuated disease course in individuals infected with HIV-1 harbouring gross genetic Nef defects (Deacon et al., 1995; Kestler et al., 1991; Kirchhoff et al., 1995). However, more subtle, naturally-occurring variation in Nef may also influence disease outcomes. For example, Nef proteins derived from HIV-1 subtype B infected elite controllers displayed relative functional impairments compared to those from progressors in the absence of major genetic defects (Mwimanzi et al., 2011c, 2013). Studies linking Nef function and disease outcomes in HIV-1 subtype C, the predominant subtype of the HIV pandemic, are however lacking.

Nef is also a highly immunogenic protein (Lichterfeld et al., 2004). Cross-sectional studies have shown that the overall breadth of Nef-specific CD8 + T cell responses does not correlate with viral control (Kiepiela et al., 2007) and in some studies a higher magnitude of these responses correlated with higher viral loads (Novitsky et al., 2003; Radebe et al., 2011). Nevertheless, the observed associations between lower viral loads and CD8 + T cell targeting of certain Nef epitopes in humans (Adland et al., 2013) and non-human primate models (Budde et al., 2012; Mudd et al., 2012), suggests that CD8 + T cell responses to specific Nef regions may be beneficial.

There is also somewhat limited evidence that immune-driven escape mutations can impair Nef function. Two HLA-B*35-associated $\mathrm{CD} 8+\mathrm{T}$ cell escape mutations in a conserved proline-rich region of Nef impaired Nef-mediated HLA class I down-regulation and virus replication when occurring in combination (Mwimanzi et al., 2011a; Ueno et al., 2008). Accumulation of novel HLA-B*57associated polymorphisms largely unique to elite controllers was associated with reduced Nef function in these individuals (Mwimanzi et al., 2013). Furthermore, a recent study predicted that over $50 \%$ of HLA-associated Nef polymorphisms would revert on transmission to an HLA-mismatched recipient, supporting biologically-relevant fitness costs of certain immune-driven mutations in Nef (Adland et al., 2013). Identification of immune-driven Nef mutations influencing its pathogenic functions could therefore reveal regions of potential relevance for inclusion in an HIV-1 vaccine designed to attenuate the infection course (Allen and Altfeld, 2008). Thus, studies to investigate this are warranted.

Also important to vaccine design is the identification of specific features of viruses that are transmitted successfully (i.e. understanding the transmission bottleneck). Transmitted/early viruses tend to exhibit particular Env characteristics, including shorter variable loops with fewer glycosylation sites (Derdeyn et al., 2004; Sagar, 2010) and near-exclusive CCR5 co-receptor usage (Sagar, 2010); however, there is little knowledge regarding whether Nef characteristics of early viruses may differ from those from later infection stages (Noviello et al., 2007). Similarly, envelope length and number of glycosylation sites may increase during the infection course (Sagar et al., 2006) (though this remains somewhat controversial (Novitsky et al., 2009a)); however, few studies have examined early sequence and/or functional changes in Nef in large numbers of patients, particularly in a subtype $C$ context (Brumme et al., 2008; Kuang et al., 2014; Noviello et al., 2007).

To address these knowledge gaps, we assessed two Nef functions implicated in pathogenesis - down-regulation of CD4 and HLA-I - in clonal plasma HIV RNA-derived Nef sequences isolated from untreated HIV-1 subtype $C$ infected individuals from different disease stages. We studied a single representative Nef clone from each patient, including 120 individuals in early infection (of whom a subset of 68 individuals were additionally characterised for Nef function one year later), and an independent group of 207 individuals in the chronic phase of infection. We investigated (i) the relationship between Nef function in early infection and subsequent rate of HIV-1 disease progression, and (ii) the relationship between Nef function and sequence, in particular immune-driven HLA-associated Nef mutations. In addition, as an exploratory analysis we assessed differences in the genetic characteristics of Nef in early and chronic infection as well as changes in Nef function and sequence over the first year of infection.

\section{Results}

\section{Nef clones: function and expression}

Nef-mediated CD4 and HLA-I down-regulation were measured for 395 Nef clones from 327 individuals (120 with acute/early infection, for whom 68 also had a follow-up clone at 1 year postinfection, as well as 207 with chronic infection) in a CEM T cell line expressing HLA-A*02:01. It should be noted that we previously observed an excellent correlation between the relative ability of Nef clones to down-regulate HLA-A*02 and HLA-B*07, justifying that HLA-A*02 down-regulation is in general representative of HLA-I down-regulation (Mann et al., 2013). The functions of patient-derived Nef sequences were expressed relative to that of the subtype B reference Nef sequence SF2, which represented $100 \%$ activity (Supplementary Table 2, representative flow plots in Fig. 1). Overall, patient-derived Nef clones showed a median CD4 down-regulation ability of 96\% (IQR, 77.6 to 99.7\%) and a median HLA-I down-regulation ability of 76\% (IQR, 53.4 to 87\%) compared to the control subtype B SF2 strain. A minority of Nef clones ( $n=47,12 \%$ ) was poorly functional ( $<45 \%$ for both Nef functions). To confirm their Nef protein expression, Western blots were performed on this subset as well a randomly-selected set of 78 Nef clones that displayed $>50 \%$ CD4 or HLA-I down-regulation ability relative to SF2 (indicating that Nef was expressed). Of the 47 poorly functional clones, 41 were not readily detectable by Western blot using polyclonal anti-Nef sera from rabbit or sheep (defined as $<30 \%$ of SF2 control, Supplementary Table 2), while 8 of the 78 functional clones were below this threshold of detection. Since we cannot exclude the possibility that Nef clones with poor expression and function are attributable to cloning or other in vitro artifact, we excluded these 41 isolates from all further analyses. This left a total of 354 Nef clones for analysis: 107 from acute/early infection, 61 from 1 year post-infection (including 56 paired clones at baseline and 1 year post-infection), and 186 from chronic infection. The Western blot band intensities of the remaining Nef clones did not correlate significantly with CD4 down-regulation (Spearman's, $r=0.16$ and $p=0.15$ ) or HLA-I down-regulation (Spearman's, $r=0.18$ and $p=0.1$ ) (Supplementary Fig. 1).

\section{Similar Nef function and sequence in different cohorts and laboratories}

Our Nef sequences from acute/early infection were derived from various cohort and clinical studies in Botswana (Tshedimoso $[n=27]$ ) and South Africa (HPP acute infection [ $n=33]$ and TRAPS $[n=47]$ cohorts). In addition, samples from the HPP acute infection cohort and Tshedimoso cohort were assayed in the HIV Pathogenesis Programme Laboratory at the University of KwaZulu-Natal while those from the TRAPS cohort were assayed in the HIV/AIDS 

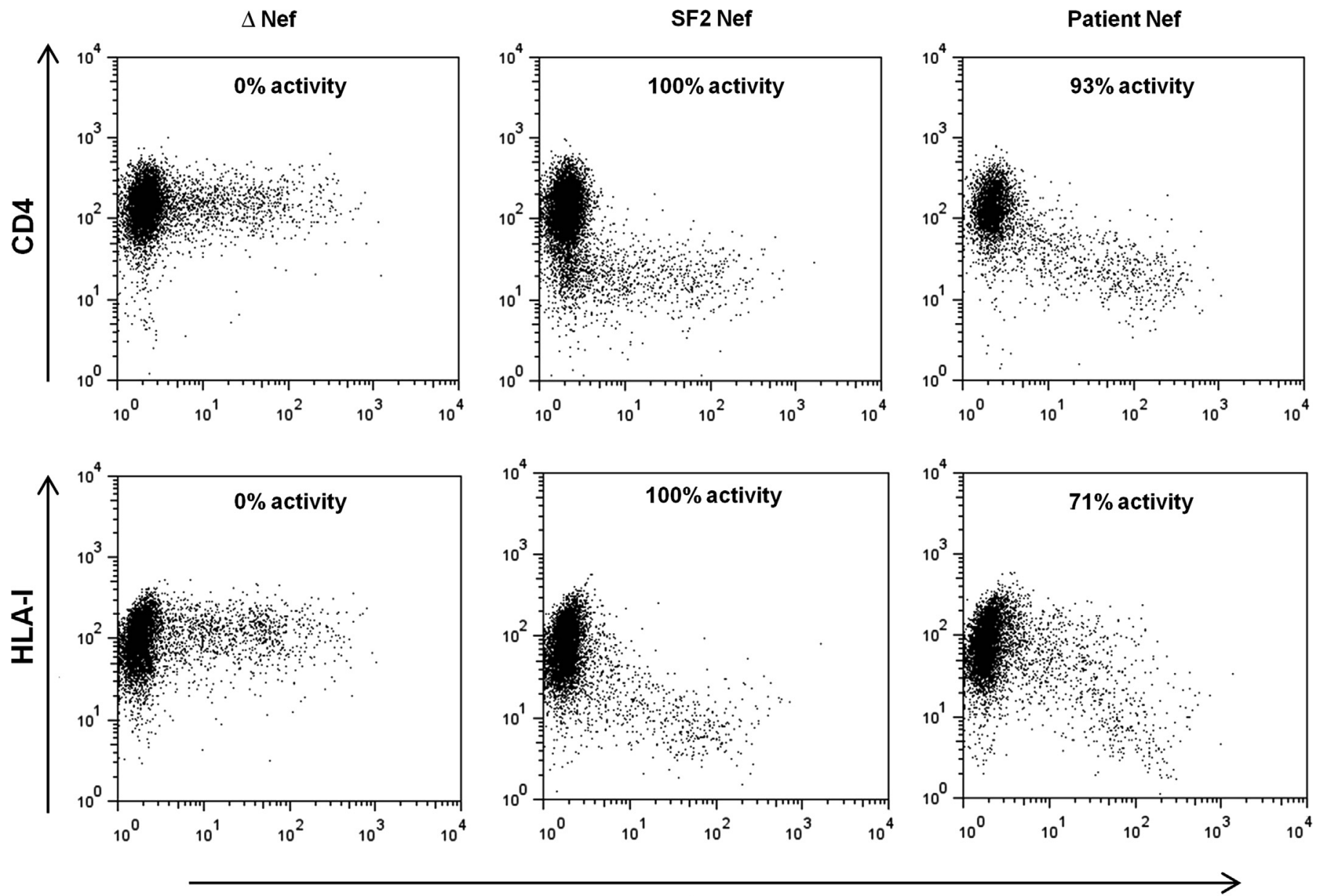

GFP

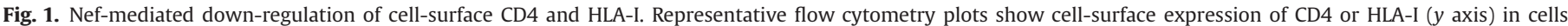

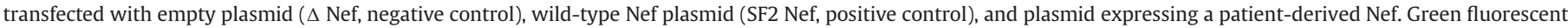
protein (GFP) expression ( $x$ axis) was used as a marker of cells transfected with plasmids.

Molecular Epidemiology Laboratory at Simon Fraser University. As such, we confirmed that there were no significant cohort- or laboratory-specific biases in Nef function before pooling data for analysis. Both Nef-mediated CD4 down-regulation and HLA-I down-regulation activities of Nef clones from recent infection were comparable across cohorts (Kruskal-Wallis, $p>0.2$ ) and laboratories (Mann-Whitney $p>0.2$ ) (Fig. 2A and B). In addition, no gross clustering of Nef clones by site was observed in a combined phylogeny (Fig. 2C), and no significant differences in the average pairwise distance between patient Nef clone and published Nef consensus $C$ sequence were observed by cohort (Kruskal-Wallis, $p=0.84$ ). Thus, all Nef sequences from acute/early infection were combined in subsequent analyses.

Association of Nef-mediated CD4 and HLA-I down-regulation function in acute/early infection with subsequent disease progression

To assess the relationship between Nef function in acute/early infection and subsequent disease progression, CD4 and HLA-I down-regulation abilities of Nef clones from the earliest timepoint post-infection were correlated with subsequent viral load set point and rate of CD4 decline. Viral load set point (median of $4.4 \log _{10}$ copies $/ \mathrm{ml}$; IQR, 3.7-5 $\log _{10}$ copies $/ \mathrm{ml}$ ) and rate of CD4 decline (median of -4 cells $/ \mathrm{mm}^{3}$ per month; IQR, -10 to -1 cells $/ \mathrm{mm}^{3}$ per month) were calculated for 102 and 107 individuals, respectively. Rate of CD4 decline was calculated over a median treatment-free follow-up of 672 days; IQR, 346-1304 days.
A trend of increased Nef-mediated CD4 down-regulation function at baseline correlating with higher viral load set point was observed (Spearman's, $r=0.19$ and $p=0.05$ ), but no relationship was observed between HLA-I down-regulation and viral load set point (Spearman's, $r=0.14$ and $p=0.18$ ) (Fig. 3A and B). Furthermore, both CD4 and HLA-I down-regulation functions were associated inversely with rate of CD4 decline, the latter significantly so (Spearman's, $r=-0.18, p=0.07$ and $r=-0.24, p=0.01$ respectively). However, baseline CD4 count and length of followup time also significantly correlated with CD4 decline in our cohorts (Spearman's, $r=-0.25, p=0.01$ and $r=0.31, p=0.001$ respectively); therefore, a multiple linear regression was performed to assess the relationship between Nef function and rate of CD4 decline while controlling for these potential confounders (see Methods and Table 1). After accounting for these and other baseline parameters such as viral load, the association between higher Nef-mediated HLA-I down-regulation and faster rate of CD4 decline remained statistically significant $(p=0.02)$ (Table 1$)$. In a multivariate analysis additionally adjusting for cohort site, HLA-I down-regulation still remained significantly associated with rate of CD4 decline ( $p=0.048$; data not shown).

Sequence-function analysis part 1: lack of genetic differences between acute/early and chronic Nef sequences

We next wished to identify sequence determinants of Nef function using a maximally-powered dataset. To do this, we first 
A

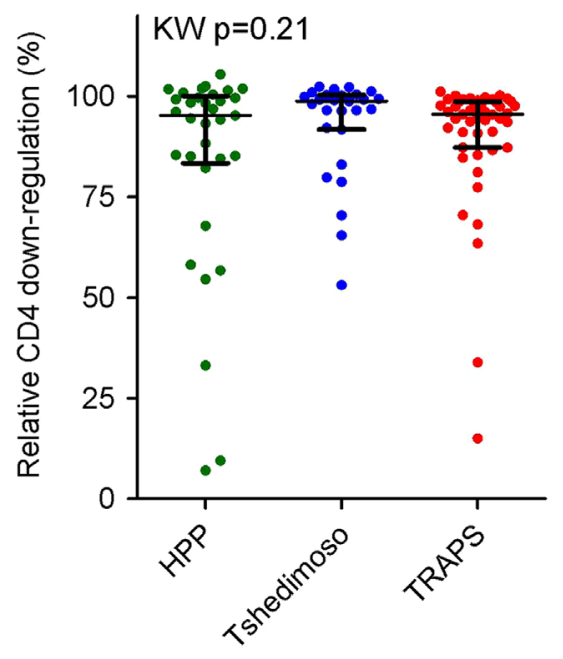

B

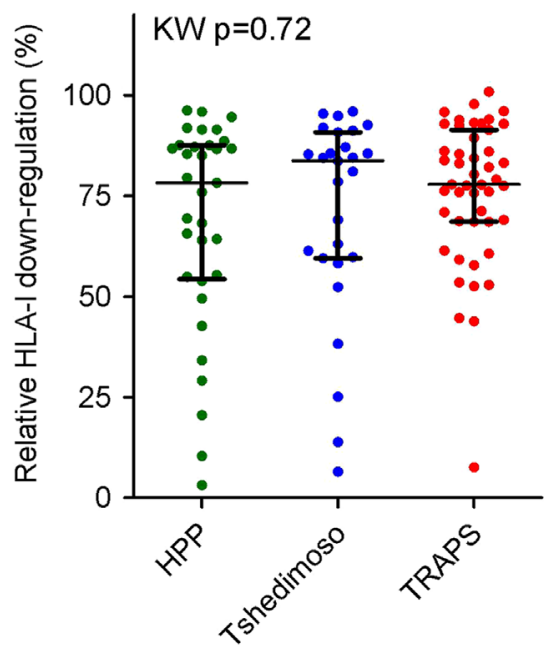

C

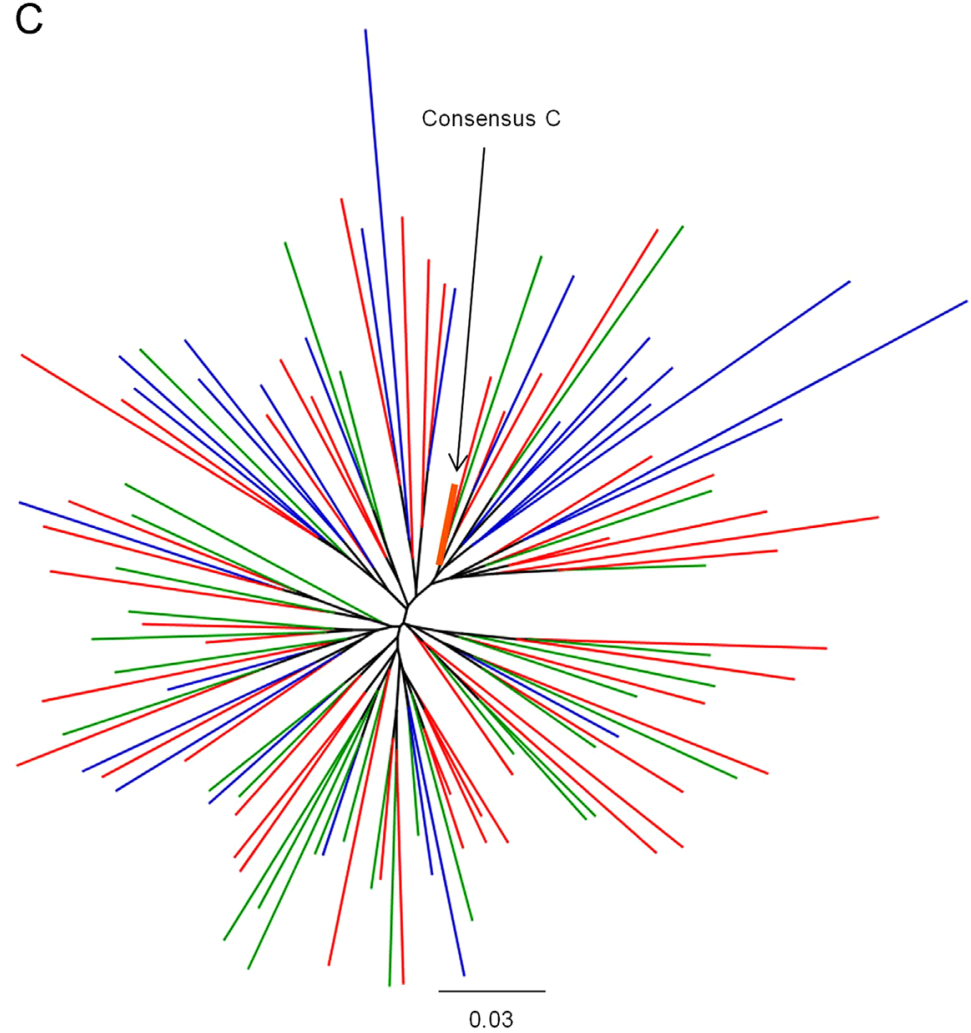

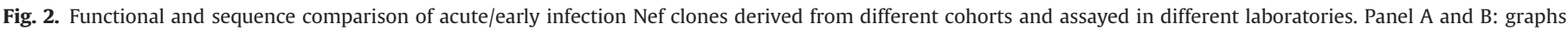

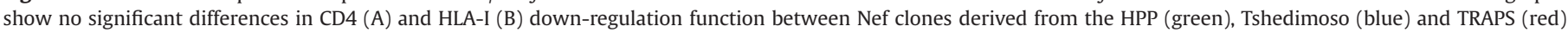

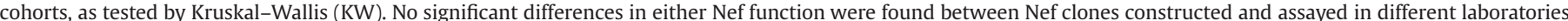

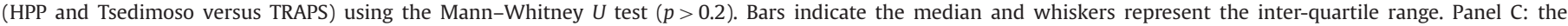

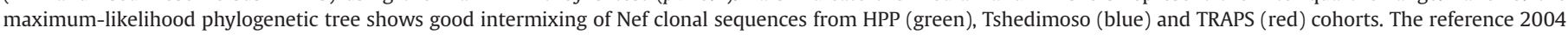
consensus C Nef sequence from the Los Alamos HIV sequence database is highlighted in orange.

compared Nef sequences from acute/early $(n=107)$ and chronic infection $(n=186)$ to investigate whether the former possess features that distinguish them from the latter and whether combining Nef sequence data across different disease stages for our sequence-function analysis would introduce major biases. No significant differences in Nef sequence length (Mann-Whitney, $p=0.35$ ) and no significant differences in the frequency of the predominant (consensus) amino acids were observed at any Nef codon between clones collected during acute/early and chronic infection (Fisher's exact test, $p>0.09$; data not shown). Moreover, acute/early and chronic sequences were interspersed in a phylogenetic tree (Fig. 4). There were, however, marginally greater pairwise distances from Nef consensus $C$ sequence in early infection clones (median $11.9 \%$ [IQR, 10.6-13.9\%] ) compared to $11.1 \%$ for chronic infection clones [IQR, 10-12.6\%], Mann-Whitney, $p=0.007$ ). In summary, although we cannot exclude the possibility that there is some transmission bottleneck in the Nef gene resulting in increased transmission of non-consensus sequences, we found overall that there were no major differences between Nef sequences from acute/early and chronic infection. Given the lack of major differences between Nef sequences from acute/early and later infection and phylogenetic inter-mixing of these groups 


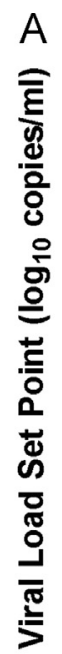
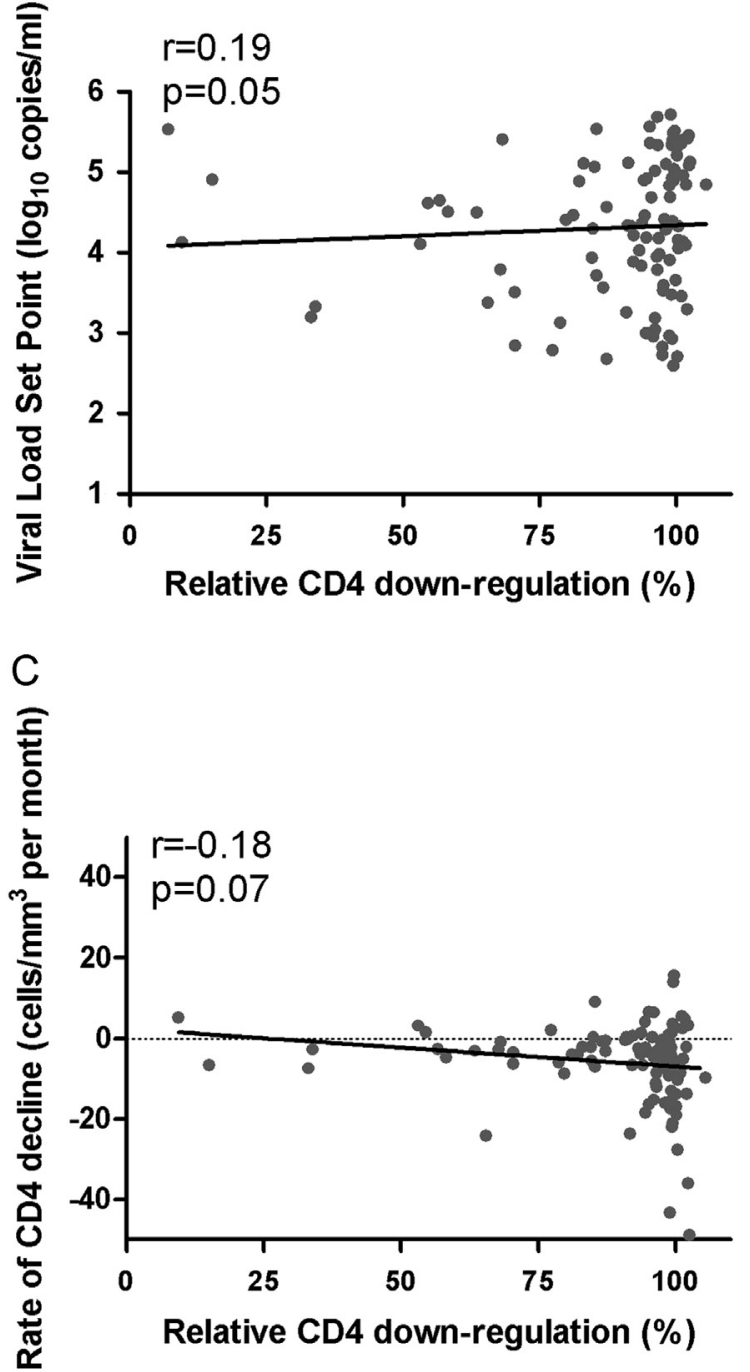

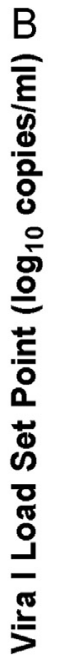

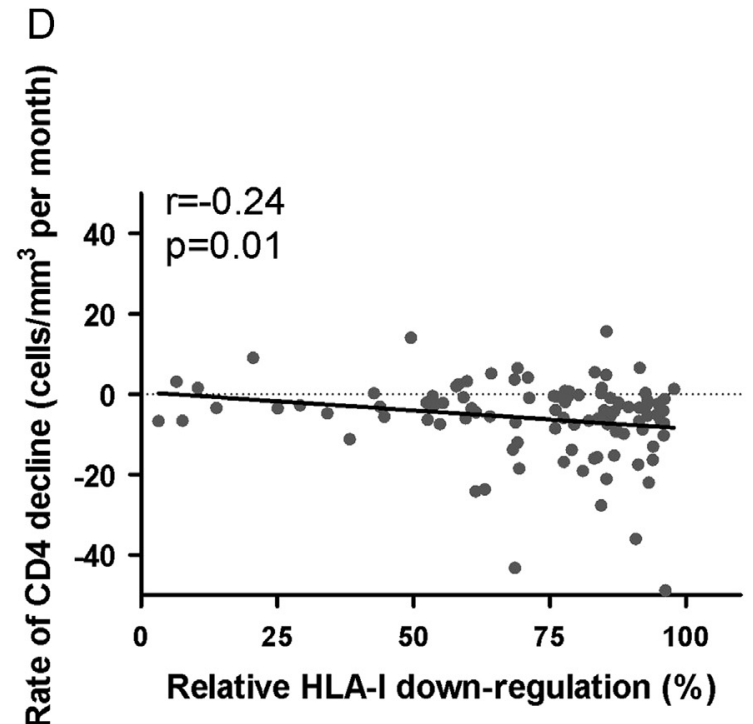

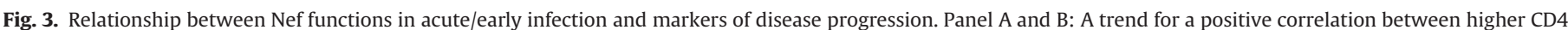

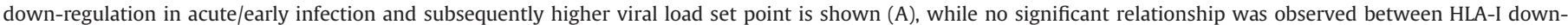

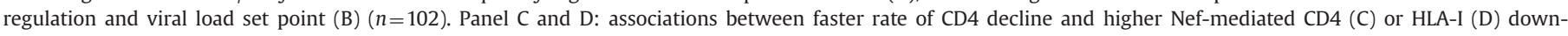

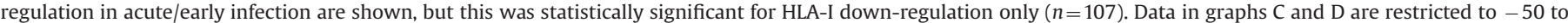
+50 cells $/ \mathrm{mm}^{3}$ per month, and thus do not include 5 outlier data points. Spearman's correlation was used to assess significance of relationships.

Table 1

Multiple linear regression models investigating the relationship between rate of CD4 decline and Nef functions.

\begin{tabular}{|c|c|c|c|c|c|}
\hline \multirow{2}{*}{ Variables } & \multicolumn{2}{|c|}{ CD4 decline, model $1^{\mathrm{a}}$} & \multirow{2}{*}{ Variables } & \multicolumn{2}{|c|}{ CD4 decline, model $2^{\mathrm{b}}$} \\
\hline & Co-efficient & $p$-value & & Co-efficient & $p$-value \\
\hline CD4 down-regulation & -7.7 & 0.12 & HLA-I down-regulation & -9.07 & 0.02 \\
\hline Square-root baseline CD4 & -1.02 & $<0.0001$ & Square-root baseline CD4 & -0.99 & $<0.0001$ \\
\hline Follow-up time & 0.01 & $<0.0001$ & Follow-up time & 0.01 & $<0.0001$ \\
\hline Log baseline viral load & -0.87 & 0.32 & Log baseline viral load & -1.04 & 0.22 \\
\hline
\end{tabular}

${ }^{\text {a }}$ Model for assessing relationship between CD4 decline and CD4 down-regulation: adjusted $r^{2}=0.25, p=<0.0001$ and $n=102$.

${ }^{\mathrm{b}}$ Model for assessing relationship between CD4 decline and HLA-I down-regulation: adjusted $r^{2}=0.28, p=<0.0001$ and $n=102$.

of sequences, we combined these into a single dataset to increase power. Therefore, subsequent sequence-function analyses were performed on a set of $n=298$ Nef clones from unique patients as follows: 107 from acute/early infection, 5 from 1 year postinfection and 186 from chronic infection (the acute/early sequence was used in the event where both acute/early and 1 year postinfection sequences were available for the same individual).
Sequence-function analysis part 2: Nef amino acids associated with increased or decreased HLA-I down-regulation function

A Nef sequence-function analysis for CD4 down-regulation identified no Nef amino acids significantly associated with variations in this Nef function. However, a similar analysis for HLA-I down-regulation identified 31 amino acid variants at 22 different 


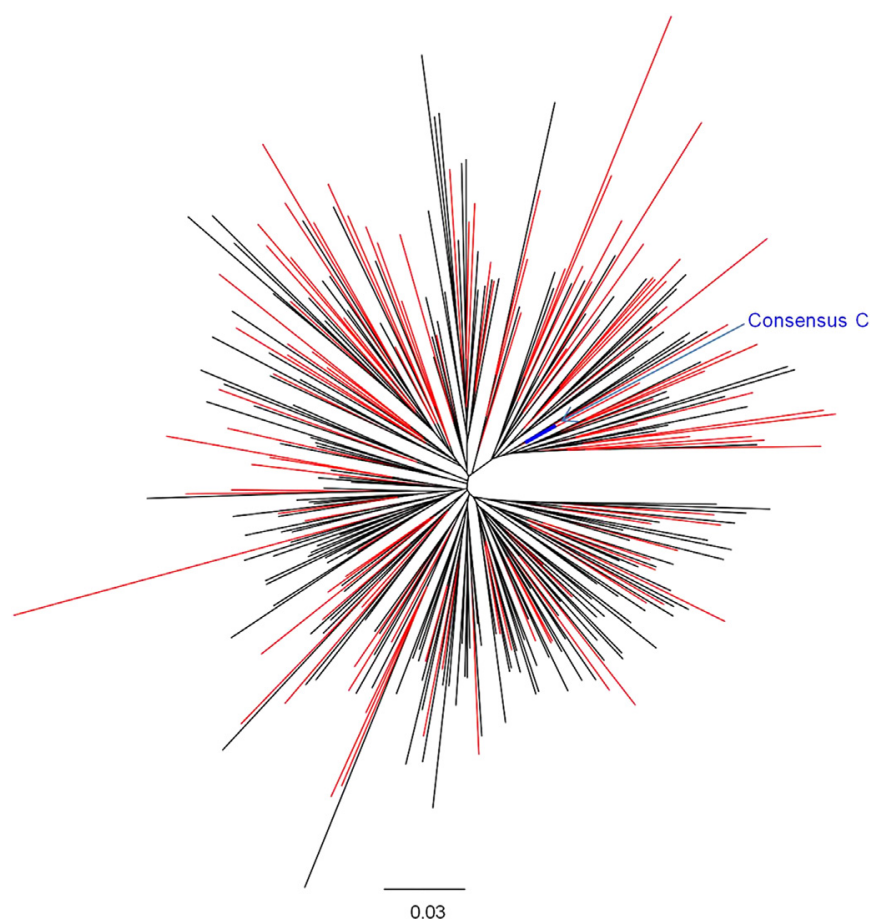

Fig. 4. Phylogenetic tree of HIV-1 subtype C Nef sequences from acute/early and chronic infection. The maximum-likelihood phylogenetic tree shows good intermixing of Nef clonal sequences derived from acute/early (red) and chronic (black) infection. The reference 2004 consensus C Nef sequence from the Los Alamos HIV sequence database is shown in blue.

codons significantly associated with this function (Table 2). Several of these associations corroborated those identified in previous studies: amino acid associations with differential HLA-I downregulation were identified at codons 3, 8, 9, 40, 102, 105, and 108 in a study of Nef subtypes A, B, C and D that included 74 subtype C Nef clonal sequences analysed in the present study (Mann et al., 2013) and similarly at codons 8 and 108 in HIV subtype B Nef sequences (Mwimanzi et al., 2013). Of note, 3 codons identified in this analysis, viz. 20, 62 and 64, are codons previously identified by mutational analysis to play a role in HLA-I down-regulation (Akari et al., 2000; Foster et al., 2011), while non-consensus variants $102 \mathrm{H}$, 105R, 108D and 199Y, associated with reduced HLA-I down-regulation (Table 2), are HLA*B44, C*07:01, B*44 and B*18, and $C^{*} 16$ associated polymorphisms respectively (Supplementary Table 1). In contrast, the consensus $108 \mathrm{E}$, which is enriched in individuals with HLA-B*57:02 (Supplementary Table 1), is linked to higher HLA-I down-regulation (Table 2). Together, these results suggest that HLAI-restricted CD8 $+\mathrm{T}$ cell responses could select polymorphisms which impact Nef-mediated HLA-I down-regulation.

Sequence-function analysis part 3: increased numbers of HLAassociated polymorphisms correlate with reduced HLA-I downregulation

To further explore the effect of HLA-associated polymorphisms on Nef function, the number of such polymorphisms present in each Nef sequence (defined according to an independently-derived reference list of HLA-associated polymorphisms in HIV subtype C; Supplementary Table 1) was correlated with Nef function (see Methods). No significant relationship between CD4 down-regulation and number of polymorphisms was observed (Spearman's, $r=0.008$ $p=0.89$ ). However, a weak trend of inverse correlation between number of HLA-associated Nef polymorphisms and HLA-I downregulation function was observed (Spearman's, $r=-0.11$ and $p=0.06$ ) (Fig. 5A).
Table 2

Amino acids $(n \geq 5)$ associated with increased or decreased Nef HLA-I down-regulation function.

\begin{tabular}{|c|c|c|c|c|c|c|c|c|}
\hline \multirow[t]{2}{*}{ Codon $^{a}$} & \multirow[t]{2}{*}{$\mathrm{AA}^{\mathrm{b}}$} & \multirow[t]{2}{*}{ Cons. $^{\mathrm{c}}$} & \multicolumn{2}{|c|}{$\begin{array}{l}\text { Relative Nef } \\
\text { function }(\%)^{\mathrm{d}}\end{array}$} & \multicolumn{2}{|c|}{ No. of samples ${ }^{\mathrm{e}}$} & \multirow[t]{2}{*}{$p$-value } & \multirow[t]{2}{*}{$\begin{array}{c}q- \\
\text { value }\end{array}$} \\
\hline & & & $\begin{array}{c}\text { With } \\
\text { AA }\end{array}$ & $\begin{array}{c}\text { Without } \\
\text { AA }\end{array}$ & $\begin{array}{c}\text { With } \\
\text { AA }\end{array}$ & $\begin{array}{l}\text { Without } \\
\text { AA }\end{array}$ & & \\
\hline 3 & $\mathrm{~N}$ & G & 85.0 & 77.8 & 32 & 266 & 0.03 & 0.4 \\
\hline 8 & $\mathrm{~S}$ & $\mathrm{~S}$ & 77.8 & 84.7 & 256 & 35 & 0.02 & 0.3 \\
\hline 9 & $\mathrm{~S}$ & $\mathrm{~S}$ & 76.2 & 90.1 & 250 & 20 & $<0.0001$ & 0.01 \\
\hline 9 & $\mathrm{R}$ & $\mathrm{S}$ & 85.5 & 77.1 & 11 & 259 & 0.007 & 0.2 \\
\hline 12 & $G$ & G & 77.0 & 84.0 & 262 & 14 & 0.01 & 0.2 \\
\hline 15 & $\mathrm{E}$ & A & 84.9 & 77.1 & 47 & 251 & 0.03 & 0.3 \\
\hline 20 & $\mathrm{M}$ & I & 83.0 & 77.6 & 88 & 210 & 0.03 & 0.4 \\
\hline 20 & I & I & 76.9 & 81.1 & 179 & 119 & 0.04 & 0.4 \\
\hline 23 & $\mathrm{~T}$ & $\mathrm{~T}$ & 76.3 & 83.9 & 186 & 112 & 0.009 & 0.2 \\
\hline 23 & A & $\mathrm{T}$ & 83.9 & 76.8 & 106 & 192 & 0.01 & 0.2 \\
\hline 40 & $\mathrm{Y}$ & $\mathrm{H}$ & 74.8 & 82.0 & 126 & 172 & 0.002 & 0.1 \\
\hline 40 & $\mathrm{H}$ & $\mathrm{H}$ & 82.1 & 74.9 & 165 & 133 & 0.003 & 0.1 \\
\hline 51 & $\mathrm{~S}$ & $\mathrm{~N}$ & 64.7 & 79.3 & 9 & 289 & 0.02 & 0.3 \\
\hline 62 & $\mathrm{E}$ & E & 79.1 & 37.7 & 293 & 5 & 0.005 & 0.2 \\
\hline 64 & $\mathrm{E}$ & E & 80.2 & 71.1 & 253 & 45 & 0.01 & 0.3 \\
\hline 64 & $\mathrm{D}$ & E & 71.1 & 79.9 & 27 & 271 & 0.02 & 0.3 \\
\hline 79 & M & M & 79.1 & 44.6 & 293 & 5 & 0.02 & 0.3 \\
\hline 102 & $\mathrm{H}$ & $\mathrm{Y}$ & 71.8 & 79.9 & 45 & 253 & 0.01 & 0.2 \\
\hline 105 & $\mathrm{R}$ & $\mathrm{K}$ & 74.1 & 80.2 & 46 & 252 & 0.03 & 0.4 \\
\hline 108 & $\mathrm{E}$ & $\mathrm{E}$ & 82.7 & 74.2 & 159 & 139 & 0.001 & 0.1 \\
\hline 108 & $\mathrm{D}$ & E & 74.3 & 82.7 & 138 & 160 & 0.002 & 0.1 \\
\hline 133 & $\mathrm{~V}$ & V & 80.2 & 68.6 & 265 & 33 & 0.005 & 0.2 \\
\hline 146 & V & V & 79.1 & 53.1 & 293 & 5 & 0.02 & 0.3 \\
\hline 156 & $\mathrm{~N}$ & A & 89.1 & 78.1 & 6 & 292 & 0.02 & 0.3 \\
\hline 161 & $\mathrm{D}$ & $\mathrm{N}$ & 84.7 & 77.0 & 53 & 244 & 0.007 & 0.2 \\
\hline 161 & $\mathrm{~N}$ & $\mathrm{~N}$ & 76.9 & 83.2 & 235 & 62 & 0.02 & 0.3 \\
\hline 178 & $\mathrm{~K}$ & $\mathrm{R}$ & 61.1 & 79.2 & 14 & 284 & 0.02 & 0.3 \\
\hline 178 & G & $\mathrm{R}$ & 84.9 & 77.9 & 40 & 258 & 0.04 & 0.4 \\
\hline 188 & $\mathrm{~N}$ & $\mathrm{~S}$ & 21.1 & 79.1 & 5 & 293 & 0.03 & 0.3 \\
\hline 199 & $\mathrm{H}$ & $\mathrm{H}$ & 79.4 & 47.8 & 290 & 8 & 0.002 & 0.1 \\
\hline 199 & $\mathrm{Y}$ & $\mathrm{H}$ & 51.8 & 79.3 & 7 & 291 & 0.005 & 0.2 \\
\hline
\end{tabular}

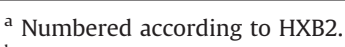

b Amino acid associated with increased or decreased HLA-I down-regulation function.

c Cohort consensus amino acid at that particular codon.

${ }^{\mathrm{d}}$ Median percentage HLA-I down-regulation function (expressed relative to SF2 control) of Nef clones with and without the amino acid.

e Number of sequences with and without the amino acid. Amino acid totals vary, as gaps in the alignment are considered missing data.

Several HLA-associated mutations in Nef have been predicted to revert upon transmission to a host lacking the original restricting HLA, suggesting they may have fitness costs (Adland et al., 2013). To investigate the relationship between such "reverting" polymorphisms and Nef HLA-I down-regulation function $(n=21$ at 15 sites previously associated with reversion, (Adland et al., 2013) and Supplementary Table 1), we repeated our analysis considering only these polymorphisms. Of note, we observed a stronger inverse correlation with HLA-I down-regulation function (Spearman's, $r=-0.21$ and $p=0.0002$ ) (Fig. 5B) compared to the original analysis undertaken on all HLAassociated polymorphisms (Fig. 5A). Collectively, these results suggest that HLA alleles can drive the selection of certain polymorphisms in Nef that impair its function.

Sequence-function analysis part 4: polymorphisms associated with HLA-B*44, but not protective alleles $B^{*} 57, B^{*} 58: 01$ and $B^{*} 81$, correlate with reduced HLA-I down-regulation

Previous studies have shown correlations between presence of polymorphisms associated with protective HLA alleles and reduced Gag-protease function (Brockman et al., 2007; Crawford et al., 2009; Schneidewind et al., 2007; Wright et al., 2011), and between increasing numbers of $B^{*} 57$-associated Nef polymorphisms largely unique to 


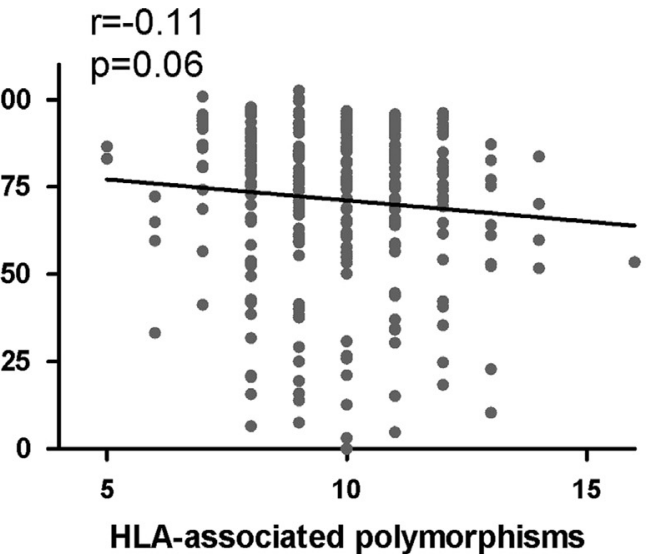

B
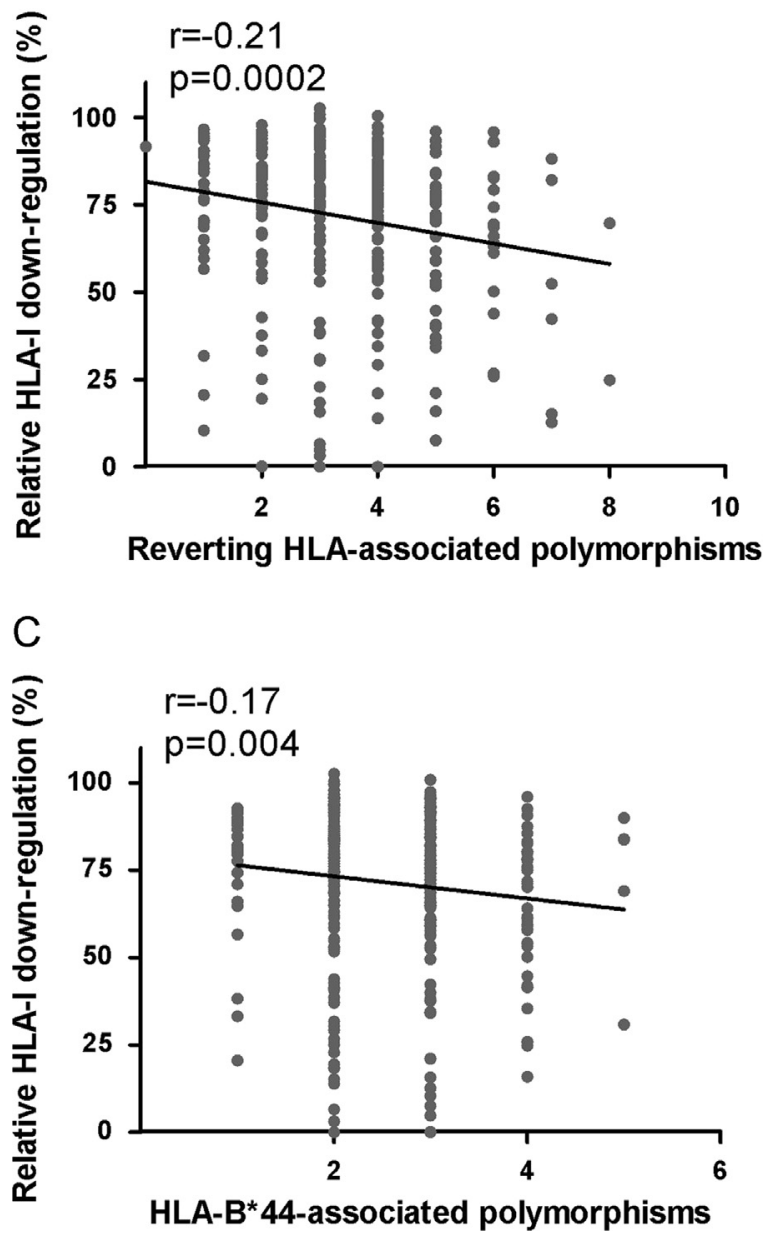

Fig. 5. Relationship between HLA-associated Nef polymorphisms and Nefmediated HLA-I down-regulation activity. Panel A: A trend for lower HLA-I down-regulation activity with increasing numbers of HLA-associated polymorphisms is shown. Panel B: A significant negative correlation between the number of non-consensus polymorphisms at HLA-associated codons previously associated with reversion (Adland et al., 2013) and HLA-I down-regulation ability is shown. Panel C: the number of HLA-B*44-associated polymorphisms present correlates negatively with HLA-I down-regulation function. Spearman's correlation was used to assess significance of relationships ( $n=298$ for all analyses).

elite controllers and reduced Nef CD4 down-regulation function (Mwimanzi et al., 2013). Therefore, we investigated whether Nef sequences harbouring polymorphisms associated with HIV subtype
C-specific protective HLA alleles B*57, B*58:01 and B*81 (Kiepiela et al., 2004) exhibited decreased Nef function. No relationship between the numbers of these polymorphisms and either Nef function was observed (Spearman's, $p>0.17$; data not shown). In fact, the number of polymorphisms specifically associated with HLA-B*57 (83G, 83E, $108 \mathrm{E}$, and $116 \mathrm{~N}$ ) was positively associated with HLA-I down-regulation function (Spearman's, $r=0.12$ and $p=0.04$ ) (data not shown). This result appeared to be driven by $108 \mathrm{E}$, the consensus amino acid at codon 108, which alone was associated with higher HLA-I downregulation (Mann-Whitney, $p=0.001$ ).

HLA-B*44:03, the most common subtype of $B^{*} 44$ in South Africa, is associated with a modest $(\approx 0.25 \log )$ reduction in viral load (Kiepiela et al., 2004; Leslie et al., 2010) and restricts a CD8+ T cell epitope (KY11, codons 92-102) within a "vulnerable" Nef region (codons 88-105) to which CD8 $+\mathrm{T}$ cell responses have been associated with a protective effect (Adland et al., 2013). There are also more Nef polymorphisms $(n=7$; 65E, 71R, 93D, $102 \mathrm{H}, 108 \mathrm{D}$ 176D, and 176V) associated with HLA-B*44 than any other allele (Supplementary Table 1), five of which are predicted to revert in the absence of HLA-B*44 (Adland et al., 2013). Consistent with a cumulative effect of HLA-B*44-driven polymorphisms on Nef function, we observed a significant inverse correlation between number of such polymorphisms present and HLA-I down-regulation function (Spearman's, $r=-0.17$ and $p=0.0042$ ) (Fig. 5C).

Nef sequence and function in the first year of infection: adaptation to host environment and lack of consistent functional change

Exploratory analyses of 56 paired acute/early and 1 year postinfection Nef clones were next performed to investigate immunedriven evolution in Nef and functional changes over the first year of infection.

Firstly, sequences of matched clones were analysed to identify common sequence changes away from or towards a particular amino acid, that were observed in $\geq 5$ individuals, between baseline and 1 year post-infection. Five such changes were identified: X83A, X102Y, $\mathrm{X} 105 \mathrm{~K}, \mathrm{E} 108 \mathrm{D}$, and $\mathrm{H} 116 \mathrm{~N}$, where $\mathrm{X}$ indicates any amino acid (Table 3); all represent codons at which HLA-associated polymorphisms have been identified (Supplementary Table 1). Changes towards consensus (X83A, X102Y, and X105K) may thus represent reversions of escape mutations, suggesting fitness costs for these (Matthews et al., 2008). Indeed, reversions at codons 83 and 102 were predicted in a recent study (Adland et al., 2013) and non-consensus amino acids at codons 83,102 and 105 have been statistically associated with reduced HLA-I down-regulation previously (Mann et al., 2013; Mwimanzi et al., 2013) and/or in the present study (Table 2). A minority of these reversions to consensus, particularly X102Y, occurred in the presence of the associated HLA allele, however such an occurrence has previously been documented (Allen et al., 2004). In contrast, HLAassociated mutations from consensus (E108D and H116N) may represent HLA-driven escape. Indeed, $\mathrm{H} 116 \mathrm{~N}$ is an HLA-B*57 escape mutation (Frater et al., 2007; Pillay et al., 2005) that has not been associated with functional costs, and 108D, which associated with decreased HLA-I down-regulation in the present and previous studies (Mann et al., 2013), was selected four out of five times in patients harbouring the associated HLA alleles B*44 and B*18. Thus, common Nef sequence changes in the first year of infection appear to be largely driven by adaptation to host HLA.

Some of these common early sequence changes are expected to increase Nef function and some are expected to decrease Nef function. As such, it is perhaps unsurprising that in a longitudinal analysis, no overall consistent changes in Nef-mediated CD4 or HLA-I down-regulation functions were observed during the first year of infection (Wilcoxon matched-pairs signed-ranks test, $p=0.54$ and $p=0.56$, respectively) (Fig. $6 \mathrm{~A}$ and $\mathrm{B}$ ). 
Table 3

Common sequence differences ( $n \geq 5$ ) occurring from acute/early to 1 year post-infection in 56 pairs of Nef clones.

\begin{tabular}{|c|c|c|c|c|}
\hline Change & Con..$^{a}$ & HLA-association $^{\text {b }}$ & HLA present ${ }^{\mathrm{C}}$ & Function association $^{\mathrm{d}}$ \\
\hline $\mathrm{X} 83 \mathrm{~A}$ & A & $\begin{array}{l}83 G-B^{*} 57, B^{*} 58: 01, A^{*} 03: 01, A^{*} 34 \\
83 E-B^{*} 57\end{array}$ & $0 / 5$ & 83G decreased HLA-I down-regulation Mann et al. (2013) \\
\hline $\mathrm{X} 102 \mathrm{Y}$ & Y & $102 \mathrm{H}-\mathrm{B}^{*} 44$ & $3 / 5$ & 102H decreased HLA-I down-regulation Mann et al. (2013) \\
\hline $\mathrm{X} 105 \mathrm{~K}$ & $\mathrm{~K}$ & $105 R-C^{*} 07: 01$ & $1 / 5$ & $\begin{array}{l}105 \mathrm{~K} \text { increased HLA-I down-regulation Mann et al. (2013) } \\
105 \text { R and Q decreased HLA-I down-regulation Mann et al. (2013) } \\
105 \text { R decreased CD4 down-regulation Mwimanzi et al. (2013) }\end{array}$ \\
\hline E108D & $\mathrm{E}$ & $\begin{array}{l}108 \mathrm{D}-\mathrm{B}^{*} 44, \mathrm{~B}^{*} 18 \\
108 \mathrm{E}-\mathrm{B}^{*} 57: 02\end{array}$ & $4 / 5$ & 108D decreased HLA-I down-regulation Mann et al. (2013), Mwimanzi et al. (2013) \\
\hline $\mathrm{H} 116 \mathrm{~N}$ & $\mathrm{H}$ & $116 N-B^{*} 57: 03$ & $0 / 5$ & No association \\
\hline
\end{tabular}

a Consensus amino acid.

${ }^{\mathrm{b}}$ HLA alleles positively associated with an amino acid at the relevant codon at $p<0.05, q<0.05$ (Supplementary Table 1).

${ }^{c}$ Indicates the number of individuals who expressed the relevant HLA allele (associated with variants at the codon at which the sequence change occurred).

d Statistical associations between Nef amino acid variants and Nef function as identified in previous studies.
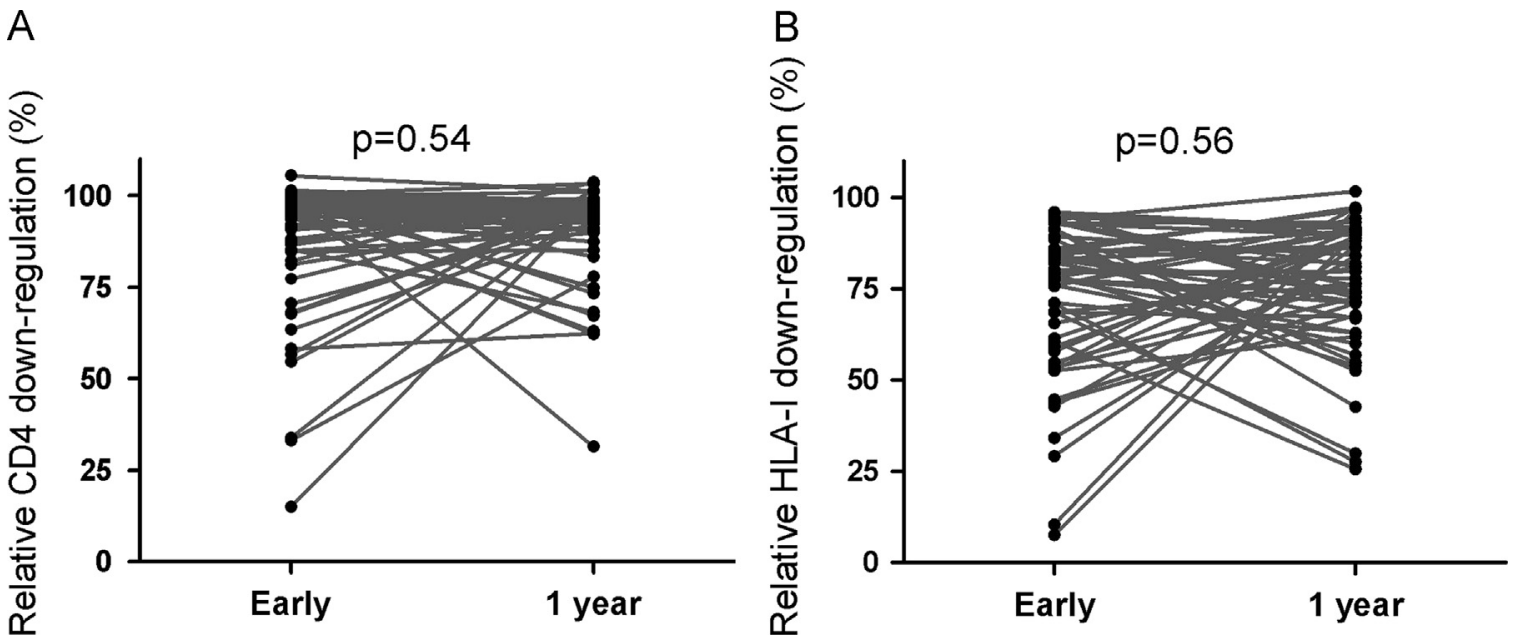

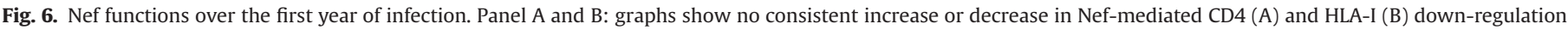

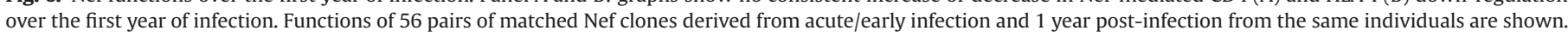
$P$ values from the Wilcoxon matched-pairs test are shown.

\section{Discussion}

There is limited direct evidence that $\mathrm{CD} 8+\mathrm{T}$ cell responses to HIV-1 Nef impose a functional cost on the virus (Adland et al., 2013; Mwimanzi et al., 2013; Ueno et al., 2008) and the contribution of Nef-specific CD8 $+\mathrm{T}$ cell responses to viral load control remains debated (Adland et al., 2013; Kiepiela et al., 2007). Furthermore, while gross genetic defects in Nef can contribute to long-term non-progression (Deacon et al., 1995; Kirchhoff et al., 1995) and attenuation of Nef functions in elite controllers have been demonstrated (Mwimanzi et al., 2013), large populationbased studies correlating Nef functions with disease progression in HIV-1 subtype C infection are lacking. Therefore, we investigated the relationships between Nef-mediated CD4 and HLA-I downregulation functions and disease progression, as well as the potential influence of immune-driven Nef sequence variation on these functions, in HIV-1 subtype C infection.

As previously observed by our group (Mann et al., 2013), Nef-mediated CD4 down-regulation function was considerably more well-conserved than HLA-I down-regulation function in our study, suggesting the former function as particularly important for the virus to conserve. This is consistent with the critical role ascribed to Nef-mediated CD4 down-regulation in mediating vira replication and pathogenesis in humanised mouse and macaque models in early infection, while Nef-mediated HLA-I downregulation is neither sufficient nor required for these pathogenic effects (Iafrate et al., 2000; Watkins et al., 2013). Despite the fairly narrow spread of observed CD4 down-regulation values in patient sequences (that could reduce our ability to identify significant correlations between this Nef function and clinical/sequence parameters), we observed a modest positive association $(p=0.05)$ between Nef-mediated CD4 down-regulation function in early infection and viral load set point. This result is consistent with reports that CD4 down-regulation directly enhances viral replication through promoting budding of infectious virions (Argañaraz et al., 2003; Lundquist et al., 2002).

On the other hand, we observed no evidence linking Nefmediated HLA-I down-regulation function in early infection and viral load set point. This is consistent with studies showing a lack of effect on SIV viral loads following knockout of HLA-I downregulation function in the macaque model (Swigut et al., 2004) and a lack of correlation between this Nef function and viral loads in a small study of HIV-1 infected individuals (Lewis et al., 2008), indicating that this function does not directly affect viral replication. However, we observed that increased Nef-mediated HLA-I down-regulation in early infection was linked with a subsequent faster rate of CD4 decline. This suggests that variability in this Nef function may affect disease progression following the initial CD4 depletion, supporting its relevance in vivo. The biological relevance of Nef-mediated HLA-I down-regulation is also supported by strong in vivo selection pressure to restore this function following mutational knock-out in an SIV model (Swigut et al., 2004). As such, the relationship between HLA-I down-regulation ability and CD4 decline may be mediated via enhanced evasion of CD8 + T cell 
responses by HLA-I down-regulation (Lewis et al., 2008; Swigut et al., 2004), though why this is not additionally manifested in higher plasma viral load remains an open question.

In this study, we found evidence that mutations selected by HLA alleles, likely $\mathrm{CD} 8+\mathrm{T}$ cell escape mutations, may reduce Nefmediated HLA-I down-regulation ability, but not CD4 downregulation function (which may be related to the highly conserved nature of that function). The presence of certain HLA-associated polymorphisms $(102 \mathrm{H}, 105 \mathrm{R}, 108 \mathrm{D}$ and $199 \mathrm{Y})$ was individually significantly associated with reduced HLA-I down-regulation. These results extend those of a previous study demonstrating a reduction in HLA-I down-regulation activity of Nef sequences harbouring a combination of escape mutations in the proline-rich region (Ueno et al., 2008). Furthermore, consistent with an inferred fitness cost of Nef HLA-associated polymorphisms associated with reversion (Adland et al., 2013); we observed that increasing numbers of these polymorphisms in our patient Nef sequences correlated strongly with reduced HLA-I down-regulation.

We additionally observed that increasing numbers of mutations associated with HLA-B*44, which has been linked to a modest reduction in viral loads (Kiepiela et al., 2004; Leslie et al., 2010), corresponded with decreased HLA-I down-regulation. Furthermore, we found that the number of polymorphisms within two overlapping peptides (codons 88-105 and 134-148), to which $\mathrm{CD} 8+\mathrm{T}$ cell responses have been associated with lower viral loads in HIV-1 infection (Adland et al., 2013), correlated significantly and negatively with HLA-I down-regulation function (data not shown). These observations suggest that functional constraints may contribute to the modest benefit associated with HLA-B* 44 and the beneficial effects of CD8 + targeting of Nef regions 88-105 and 134-148, either directly (via fitness-costly escape) or indirectly (via sustained $\mathrm{CD} 8+\mathrm{T}$ cell responses to a mutationally constrained region i.e. delayed escape). In fact, there is much evidence that a balance between these two factors influences viral load (Crawford et al., 2009; Kawada et al., 2006; Wright et al., 2011). Interestingly, we found no evidence that Nef polymorphisms commonly associated with the protective HLA-B*57 allele reduce Nef function, while others have found that the number of non-canonical HLAB*57-associated Nef polymorphisms particular to elite controllers, but not those commonly associated with $B * 57$, correlated negatively with several Nef functions in elite-controller-derived Nef sequences (Mwimanzi et al., 2013). Nevertheless, it is possible that HLA-B*57 responses to Nef epitopes may contribute to slower progression in $B^{*} 57$ individuals who are not elite controllers, considering that the $B^{*} 57$-restricted Nef epitopes overlap considerably with Nef regions associated with viral control (Adland et al., 2013) and that maintenance of Nef HW9 responses was associated with long-term non-progression in HLA-B*57-expressing individuals (Navis et al., 2008).

Of relevance to HIV vaccine design is whether viruses in early infection differ from those in chronic infection (which might indicate preferential transmission of certain variants). Although $\mathrm{Nef}$ sequences from early infection were marginally less similar to the consensus $\mathrm{C}$ sequence, we found no features that clearly distinguished early from chronic Nef sequences. This is consistent with a previous report describing similar diversity in donor and acutelyinfected recipient Nef sequences as well as a lack of optimisation of specific Nef functions during transmission (Noviello et al., 2007). Our longitudinal analysis of individuals from early to 1 year post-infection revealed no consistent functional changes and that common sequence changes occurring during this period were reversions and selections of HLA-associated mutations. Similarly, it was previously found that the differences between HIV sequences from early and later infection stages largely represented reversion of mutations and escape mutations occurring post-transmission in adaptation to the specific host environment (Treurnicht et al., 2010). Also in line with these findings, Noviello et al. (2007) observed that CD8+ T cell responses appeared to be a major driver of Nef evolution during early HIV-1 infection (Noviello et al., 2007).

In summary, this study provides evidence that variability in Nef-mediated CD4 and HLA-I down-regulation ability among subtype $C$ viruses in the absence of obvious Nef genetic defects may influence disease progression. Furthermore, immune pressure on certain regions of Nef may result in selection of mutations that reduce the HLA-I down-regulation function of Nef. Together with previous studies showing clinical benefit associated with CD8 $+\mathrm{T}$ cell responses to particular epitopes in Nef, results support certain Nef epitopes for consideration as components of an HIV vaccine designed to attenuate the infection course.

\section{Methods}

\section{Study subjects}

Antiretroviral naïve patients with acute/early and chronic HIV-1 subtype $C$ infection were recruited from established observational cohorts and clinical trial sites in South Africa and Botswana.

Study participants with acute/early infection $(n=120)$ comprised 39 individuals from the HIV Pathogenesis Programme (HPP) Acute Infection Cohort in Durban, South Africa (Radebe et al., 2011; Wright et al., 2011), 31 from the Tshedimoso Study in Botswana (Novitsky et al., 2009b, 2009c; Wright et al., 2011), and 50 participants of the Tenofovir Gel Research for AIDS Prevention Science (TRAPS) Cohort (in KwaZulu-Natal, South Africa) who seroconverted in the CAPRISA 004 trial and were from the placebo arm (Abdool Karim et al., 2010; Chopera et al., 2013). For acute/early patients, plasma samples used were collected at a median of 49 days post-infection (interquartile range [IQR], 33-66 days post-infection). For 68 of these patients, for whom plasma was available, an additional plasma sample collected at a median of 374 days post-infection [IQR, 363-381 days post-infection] was analysed. Viral load, CD4 count and high resolution HLA class I data were available for all study participants with acute/early infection. Study participants with chronic infection $(n=207)$ were from the Sinikithemba Cohort in Durban, South Africa (Kiepiela et al., 2004; Mann et al., 2013; Wright et al., 2010). Viral loads, CD4 counts, and high resolution HLA class I data were available for these chronically infected patients as previously described (Kiepiela et al., 2004).

Written informed consent was obtained from all study participants and ethical approval was obtained from the relevant institutional committees.

\section{Generation and sequencing of Nef clones}

A single Nef clone per study participant was analysed. A subset of the Nef clones studied here were previously published, including 85 from HIV-1 subtype C chronically infected individuals (Mann et al., 2013) and 48 from individuals in acute/early infection who were in the placebo arm of the CAPRISA 004 trial (Chopera et al., 2013). Nef clones were generated from plasma and sequenced as previously described (Mann et al., 2013) Briefly, nef was amplified from plasma-derived HIV-1 RNA using primers containing AscI and SacII restriction enzyme sites. The nef gene was cloned into a pSELECT-GFPzeo expression plasmid (Invivogen, San Diego, CA, USA), which was engineered with the corresponding restriction enzyme sites and had separate promoters for the nef gene and green fluorescent protein (GFP) expression. Bulk nef polymerase chain reaction (PCR) products as well as nef clonal sequences were sequenced using the ABI Prism Big Dye Terminator v3.1 Cycle Sequencing Kit (Applied Biosystems, Foster City, CA, USA). For each study participant, a single representative Nef clone with an 
open reading frame and that clustered with the corresponding bulk sequence in a phylogenetic tree (Guindon and Gascuel, 2003) was selected for CD4 and HLA-I down-regulation analyses. Nef sequences were aligned to HXB2 and stripped of insertions with respect to HXB2 using HyPhy (Kosakovsky Pond et al., 2005). All nef clones were confirmed to be HIV-1 subtype C using the recombinant identification program (RIP; http://www.hiv.lanl.gov/content/sequence/RIP/RIP. html). Maximum-likelihood phylogenetic trees were constructed using Phyml (Guindon and Gascuel, 2003) and pairwise genetic distances from the 2004 consensus C Nef sequence (available at http://www.hiv. lanl.gov/content/sequence/NEWALIGN/align.html) were calculated using PATRISTIC (Fourment and Gibbs, 2006). VESPA (http://www. hiv.lanl.gov/content/sequence/VESPA/vespa.html) was used to identify signature differences in consensus amino acids between groups of sequences. Sequences analysed in this study are available under Genbank accession numbers KF208819, KF208821-3, KF208825-8, KF208831-4, KF208836, KF208838-9, KF208842-3, KF208845, KF208847-208853, KF208855, KF208857-208861, KF208863-5, KF208867, KF208870, KF208872-3, KF208878-9, KF208886, KF208889, KF208893-5, KC906734-906805, KC906991-2, and KM262907-263141.

\section{CD4 and HLA-I down-regulation}

The CD4 and HLA-I down-regulation activities of Nef clones were measured as previously described using a CEM-derived T cell line expressing CD4 and engineered to stably express HLA-A*02:01 (Mann et al., 2013). Briefly, 300,000 cells were electroporated at $250 \mathrm{~V}$ and $950 \mu \mathrm{F}$ with $4 \mu \mathrm{g}$ Nef clone and incubated for $20-24 \mathrm{~h}$, followed by staining using APC-labelled anti-CD4 and PE-labelled anti-HLA-A*02 antibodies (BD Biosciences, San Jose, CA, USA). CD4 and HLA-I cell surface expression was measured by flow cytometry in successfully transfected (i.e GFP-expressing) cells. The CD4 and HLA-I down-regulation activities (indicated by median fluorescence intensity [MFI] of CD4/HLA-I expression) of Nef clones were expressed relative to that of the positive control (SF2 Nef, set at $100 \%$ function) and the negative control (empty pSELECT plasmid,

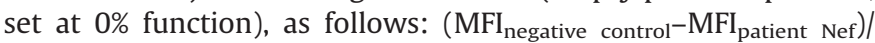
$\left(\mathrm{MFI}_{\text {negative control }}-\mathrm{MFI}_{\text {positive control }}\right)$. For the majority of Nef clones $(n=297)$, a single transfection per Nef clone was performed and stained in duplicate, with duplicate measurements in excellent agreement (Spearman's, $r=0.98$ and $p<0.0001$ for both Nef functions). For a subset of Nef clones $(n=98)$, duplicate independent transfections were performed and also correlated well (Spearman's, $r=0.8$ and $p<0.0001$ for CD4 down-regulation and $r=0.9$ and $p<0.0001$ for HLA-I down-regulation). Averaged results were analysed.

\section{Nef expression}

A minority (47 of $395,12 \%$ ) of Nef clones exhibited functions $<45 \%$ of that of the reference strain SF2 for both CD4 and HLA-I down-regulation. For these, as well as a random selection of 78 Nef clones with functions above this threshold, Nef protein levels were measured by Western blot as previously described (Mann et al., 2013; Mwimanzi et al., 2011b). Briefly, following transfection of 1 million CEM cells with $10 \mu \mathrm{g}$ of Nef clones and a $24 \mathrm{~h}$ incubation, cells were lysed. Following SDS-PAGE of cell lysates, proteins were electroblotted onto a polyvinylidene difluoride membrane and the presence of Nef protein was detected using rabbit polyclonal antiHIV-1 Nef primary antibody (NIH AIDS Research and Reference Reagent Program, USA) and horseradish peroxidase (HRP)-conjugated donkey anti-rabbit IgG secondary antibody (Amersham Biosciences, Little Chalfont, UK). Samples that failed Nef detection with the rabbit antibody were next probed using sheep polyclonal anti-HIV-1 Nef primary antibody (NIBSC Center for AIDS Reagents,
UK) and HRP donkey anti-sheep IgG secondary antibody (Jackson ImmunoResearch Europe Ltd, Suffolk, UK). Actin expression was quantified simultaneously using monoclonal mouse anti-actin primary antibody (Sigma, St. Louis, Missouri, USA) and HRP goat anti-mouse secondary antibody (Jackson ImmunoResearch Europe Ltd, Suffolk, UK). Chemiluminescence of bands was measured using ImageQuant LAS 4000 (GE Healthcare Life Sciences, Little Chalfont, UK). All Nef bands, including that of the SF2 control, were quantified relative to actin expression. These values were then normalised to that of SF2 to obtain percentage expression.

\section{Definition of HLA-associated polymorphisms in HIV-1 subtype C Nef}

HLA-associated polymorphisms are HIV amino acid variants that are significantly over- (or under) represented in the presence of a particular HLA allele, as identified in statistical association studies of linked host (HLA) and HIV sequence datasets. A comprehensive list of HLA-associated polymorphisms was previously generated based on 1336 subtype C Nef sequences from individuals from southern Africa using methods correcting for HIV phylogeny, amino acid co-variation, and HLA linkage disequilibrium (Carlson et al., 2014). HIV Nef polymorphisms in this list that were significantly positively associated with the expression of a particular HLA allele at $q<0.05$ were defined as "HLA-associated polymorphisms" in the present analysis (Supplementary Table 1).

The total number of HLA-associated polymorphisms was counted in each Nef clonal sequence. This was done without taking into account the HLA alleles expressed by the patient, since the aim of this analysis was to investigate the relationship between number of HLA-associated polymorphisms in Nef (irrespective of whether they were transmitted or selected in the present host) and Nef function.

This analysis was repeated by summing only the subset of HLAassociated polymorphisms inferred to be "reverting" (defined as those where the absence of the restricting HLA allele is significantly associated with over-representation of the consensus amino acid or under-representation of the polymorphism in statistical association studies), suggesting that they confer a fitness cost. In this analysis, non-consensus polymorphisms at sites previously defined as "reverting" ((Adland et al., 2013), listed in Supplementary Table 1), were counted in each Nef clonal sequence.

\section{Statistical analysis}

\section{Nef function versus disease progression analyses}

Nef-mediated CD4 and HLA-I down-regulation functions in acute/early infection were correlated with markers of subsequent disease progression, namely viral load set point and rate of CD4 decline, using Spearman's correlation. Viral load set point was calculated as the average viral load from 3 to 12 months postinfection. Simple linear regression was used to compute the rate of CD4 count decline for each individual over the treatment-free follow up period, where rate of decline was defined as the estimated slope of the fitted regression line (Wright et al., 2010). Multiple linear regression was also performed to assess the relationship between Nef functions and rate of CD4 decline while controlling for baseline CD4 count and length of follow-up. The nature of the CD4 decline data (a mixture of positive and negative values) prevented the use of traditional data transformations (e.g. square root, log, and inverse transformations). We thus limited the analysis to CD4 decline values within the range of -50 to 50 cells/ $\mathrm{mm}^{3}$ per month since this ensured that the assumptions of normality were sufficiently met and included $>95 \%$ of the data. Upon fitting the final model we tested the assumptions of normally distributed residuals using the Shapiro-Wilk test, and 
validated that there was no relationship between the residuals and fitted values using Spearman's correlation.

\section{Nef sequence-function analyses}

Comparisons of Nef sequence and/or Nef function were assessed using the Mann-Whitney $U$-test (with the exception of patients for whom paired early versus 1-year Nef clones were compared, in which case the Wilcoxon matched-pairs test was used), and Spearman's correlation as appropriate. Significant differences in cohort consensus amino acids between acute/early and chronic Nef sequences were identified using Fisher's exact test. Unless indicated otherwise, the significance cut-off for all statistical analyses was $p<0.05$. Specific Nef amino acids (present at a frequency of $n \geq 5$ in our dataset) associated with differences CD4 or HLA-I down-regulation function were assessed using the Mann-Whitney $U$ test. Multiple comparisons were addressed using $q$-values, the $p$-value analogue of the false-discovery rate (Storey and Tibshirani, 2003). Here, associations with $p<0.05$ and $q \leq 0.4$ are reported.

\section{Acknowledgements}

This research was funded by the NIH (R37AI067073, RO1AI057027) and the International AIDS Vaccine Initiative (UKZNRSA1001). JKM received a pilot grant from the Canada-Sub Saharan Africa (CANSSA) HIV/AIDS Network, funded by the Global Health Research Initiative, a collaborative partnership of the Canadian Institutes of Health Research (CIHR), the Canadian International Development Agency, and the International Development Research Centre (project 106355-001). DRC is a recipient of the Canada-HOPE fellowship from the Canadian Institutes for Health Research (CIHR) and Sanofi-Aventis, and fellowships from the Clinical Infectious Diseases Research Initiative Fellowship and the Claude Leon Foundation, South Africa. XTK is funded by a CAHR/BMS Master's Scholarship in Basic Science. AQL is funded by a CIHR Frederick Banting and Charles Best Masters Award. RD is supported by the Merck-Canada Training of Aboriginal Youth in Biomedical Labs program at Simon Fraser University. PM is the recipient of Postdoctoral fellowships from the Michael Smith Foundation for Health Research (MSFHR; Canada) and the CIHR. MAB holds a Canada Research Chair, Tier 2, in Viral Pathogenesis and Immunity. ZLB is the recipient of a CIHR New Investigator Award and a Scholar Award from the MSFHR. TN holds the South African DST/NRF Research Chair in Systems Biology of HIV/AIDS, the Victor Daitz Chair in HIV/TB Research and an International Early Career Scientist Award from the Howard Hughes Medical Institute.

We thank Dr. Bemuluyigza Baraki for technical assistance; Dr. Johannes Viljoen and the Africa Centre laboratory for providing access to tissue culture and sequencing facilities; and all study participants and support staff.

\section{Appendix A. Supporting information}

Supplementary data associated with this article can be found in the online version at http://dx.doi.org/10.1016/j.virol.2014.08.009.

\section{References}

Abdool Karim, Q., Abdool Karim, S.S., Frohlich, J.A., Grobler, A.C., Baxter, C., Mansoor, L.E., Kharsany, A.B., Sibeko, S., Mlisana, K.P., Omar, Z., Gengiah, T.N. Maarschalk, S., Arulappan, N., Mlotshwa, M., Morris, L., Taylor, D., CAPRISA 004 Trial Group, 2010. Effectiveness and safety of tenofovir gel, an antiretroviral microbicide, for the prevention of HIV infection in women. Science 329 (5996), 1168-1174.
Adland, E., Carlson, J.M., Paioni, P., Kløverpris, H., Shapiro, R., Ogwu, A., Riddell, L., Luzzi, G., Chen, F., Balachandran, T., Heckerman, D., Stryhn, A., Edwards, A. Ndung'u, T., Walker, B.D., Buus, S., Goulder, P., Matthews, P.C., 2013. Nef-specific CD8 + T cell responses contribute to HIV-1 immune control. PLoS One 8 (9) e73117 (doi: 73110.71371/journal.pone.0073117).

Akari, H., Arold, S., Fukumori, T., Okazaki, T., Strebel, K., Adachi, A., 2000. Nefinduced major histocompatibility complex class I down-regulation is functionally dissociated from its virion incorporation, enhancement of viral infectivity, and CD4 down-regulation. J. Virol. 74 (6), 2907-2912.

Allen, T., Altfeld, M., Yu, X.G., O’Sullivan, K.M., Lichterfeld, M., Le Gall, S., John, M., Mothe, B.R., Lee, P.K., Kalife, E.T., Cohen, D.E., Freedberg, K.A., Strick, D.A. Johnston, M.N., Sette, A., Rosenberg, E., Mallal, S.A., Goulder, P.J.R., Brander, C. Walker, B.D., 2004. Selection, transmission, and reversion of an antigenprocessing cytotoxic T-lymphocyte escape mutation in human immunodeficiency virus type 1 infection. J. Virol. 78 (13), 7069-7078.

Allen, T.M., Altfeld, M., 2008. Crippling HIV one mutation at a time. J. Exp. Med. 205 (5), 1003-1007.

Argañaraz, E.R., Schindler, M., Kirchhoff, F., Cortes, M.J., Lama, J., 2003. Enhanced CD4 down-modulation by late stage HIV-1 nef alleles is associated with increased Env incorporation and viral replication. J. Biol. Chem. 278 (36), 33912-33919.

Brockman, M.A., Schneidewind, A., Lahaie, M., Schmidt, A., Miura, T., DeSouza, I., Ryvkin, F., Derdeyn, C.A., Allen, S., Hunter, E., Mulenga, J., Goepfert, P.A., Walker, B.D., Allen, T.M., 2007. Escape and compensation from early HLA-B57-mediated cytotoxic T-lymphocyte pressure on human immunodeficiency virus type $1 \mathrm{Gag}$ alter capsid interactions with cyclophilin A. J. Virol. 81 (22), 12608-12618.

Brumme, Z.L., Brumme, C.J., Carlson, J., Streeck, H., John, M., Eichbaum, Q., Block, B. L., Baker, B., Kadie, C., Markowitz, M., Jessen, H., Kelleher, A.D., Rosenberg, E., Kaldor, J., Yuki, Y., Carrington, M., Allen, T.M., Mallal, S., Altfeld, M., Heckerman, D. Walker, B.D., 2008. Marked epitope- and allele-specific differences in rates of mutation in human immunodeficiency type 1 (HIV-1) Gag, Pol, and Nef cytotoxic T-lymphocyte epitopes in acute/early HIV-1 infection. J. Virol. 82 (18), 9216-9227.

Budde, M.L., Greene, J.M., Chin, E.N., Ericsen, A.J., Scarlotta, M., Cain, B.T., Pham, N.H., Becker, E.A., Harris, M., Weinfurter, J.T., O'Connor, S.L., Piatak, M.J., Lifson, J.D. Gostick, E., Price, D.A., Friedrich, T.C., O'Connor, D.H., 2012. Specific CD8+ T cell responses correlate with control of simian immunodeficiency virus replication in Mauritian cynomolgus macaques. J. Virol. 86 (14), 7596-7604.

Carlson, J.M., Schaefer, M., Monaco, D.C., Batorsky, R., Claiborne, D.T., Prince, J. Deymier, M.J., Ende, Z.S., Klatt, N.R., DeZiel, C.E., Lin, T.H., Peng, J., Seese, A.M., Shapiro, R., Frater, J., Ndung'u, T., Tang, J., Goepfert, P., Gilmour, J., Price, M.A Kilembe, W., Heckerman, D., Goulder, P.J., Allen, T.M., Allen, S., Hunter, E., 2014 HIV transmission. Selection bias at the heterosexual HIV-1 transmission bottleneck. Science 345 (6193), 1254031 (doi: 1254010.1251126/science.1254031).

Chopera, D.R., Mann, J.K., Mwimanzi, P., Omarjee, S., Kuang, X.T., Ndabambi, N. Goodier, S., Martin, E., Naranbhai, V., Abdool-Karim, S., Abdool-Karim, Q. Brumme, Z.L., Ndung'u, T., Williamson, C., Brockman, M.A., and the CAPRISA 004 TRAPS Team, 2013. No evidence for selection of HIV-1 with enhanced GagProtease or Nef function among breakthrough infections in the CAPRISA 004 tenofovir microbicide trial. PLoS One 8 (8), e71758 (doi: 71710.71371/journal. pone.0071758).

Crawford, H., Lumm, W., Leslie, A., Schaefer, M., Boeras, D., Prado, J.G., Tang, J., Farmer, P., Ndung'u, T., Lakhi, S., Gilmour, J., Goepfert, P., Walker, B.D., Kaslow R., Mulenga, J., Allen, S., Goulder, P.J.R., Hunter, E., 2009. Evolution of HLAB*5703 HIV-1 escape mutations in HLA-B*5703-positive individuals and their transmission recipients. J. Exp. Med. 206, 909-919.

Deacon, N.J., Tsykin, A., Solomon, A., Smith, K., Ludford-Menting, M., Hooker, D.J., McPhee, D.A., Greenway, A.L., Ellett, A., Chatfield, C., Lawson, V.A., Crowe, S. Maerz, A., Sonza, S., Learmont, J., Sullivan, J.S., Cunningham, A., Dwyer, D. Dowton, D., Mills, J., 1995. Genomic structure of an attenuated quasi species of HIV-1 from a blood transfusion donor and recipients. Science 270 (5238), 988-991.

Derdeyn, C.A., Decker, J.M., Bibollet-Ruche, F., Mokili, J.L., Muldoon, M., Denham, S A., Heil, M.L., Kasolo, F., Musonda, R., Hahn, B.H., Shaw, G.M., Korber, B.T., Allen, S., Hunter, E., 2004. Envelope-constrained neutralization-sensitive HIV-1 after heterosexual transmission. Science 303, 2019-2022.

Foster, J.L., Denial, S.J., Temple, B.R.S., Garcia, J.V., 2011. Mechanisms of HIV-1 Nef function and intracellular signaling. J. Neuroimmune Pharmacol. 6, 230-246.

Foster, J.L., Garcia, J.V., 2008. HIV-1 Nef: at the crossroads. Retrovirology 5, 84. http: //dx.doi.org/10.1186/1742-4690-1185-1184.

Fourment, M., Gibbs, M.J., 2006. PATRISTIC: a program for calculating patristic distances and graphically comparing the components of genetic change. BMC Evol. Biol. 6, 1 .

Frater, A.J., Brown, H., Oxenius, A., Günthard, H.F., Hirschel, B., Robinson, N., Leslie, A.J., Payne, R., Crawford, H., Prendergast, A., Brander, C., Kiepiela, P., Walker, B. D., Goulder, P.J.R., McLean, A., Phillips, R.E., Swiss HIV-Cohort Study, 2007 Effective T-cell responses select human immunodeficiency virus mutants and slow disease progression. J. Virol. 81 (12), 6742-6751.

Guindon, S., Gascuel, O., 2003. A simple, fast, and accurate algorithm to estimate large phylogenies by maximum likelihood. Syst. Biol. 52 (5), 696-704.

Iafrate, A.J., Carl, S., Bronson, S., Stahl-Hennig, C., Swigut, T., Skowronski, J., Kirchhoff, F., 2000. Disrupting surfaces of nef required for downregulation of CD4 and for enhancement of virion infectivity attenuates simian immunodeficiency virus replication in vivo. J. Virol. 74 (21), 9836-9844.

Kawada, M., Igarashi, H., Takeda, A., Tsukamoto, T., Yamamoto, H., Dohki, S. Takiguchi, M., Matano, T., 2006. Involvement of multiple epitope-specific 
cytotoxic T-lymphocyte responses in vaccine-based control of simian immunodeficiency virus replication in rhesus macaques. J. Virol. 80 (4), 1949-1958.

Kestler, H.W., Ringler, D.J., Mori, K., Panicali, D.L., Sehgal, P.K., Daniel, M.D. Desrosiers, R.C., 1991. Importance of the nef gene for maintenance of high virus loads and for development of AIDS. Cell 65 (4), 651-662.

Kiepiela, P., Leslie, A.J., Honeyborne, I., Ramduth, D., Thobakgale, C., Chetty, S. Rathnavalu, P., Moore, C., Pfafferott, K.J., Hilton, L., Zimbwa, P., Moore, S., Allen, T., Brander, C., Addo, M.M., Altfeld, M., James, I., Mallal, S., Bunce, M., Barber, L D., Szinger, J., Day, C., Klenerman, P., Mullins, J., Korber, B., Coovadia, H.M., Walker, B.D., Goulder, P.J.R., 2004. Dominant influence of HLA-B in mediating the potential co-evolution of HIV and HLA. Nature 432, 769-774.

Kiepiela, P., Ngumbela, K., Thobakgale, C., Ramduth, D., Honeyborne, I., Moodley, E., Reddy, S., de Pierres, C., Mncube, Z., Mkhwanazi, N., Bishop, K., van der Stok, M. Nair, K., Khan, N., Crawford, H., Payne, R., Leslie, A., Prado, J., Prendergast, A. Frater, J., McCarthy, N., Brander, C., Learn, G.H., Nickle, D., Rousseau, C., Coovadia, H., Mullins, J.I., Heckerman, D., Walker, B.D., Goulder, P, 2007. CD8 + T-cell responses to different HIV proteins have discordant associations with viral load. Nat. Med. 13 (1), 46-53.

Kirchhoff, F., Greenough, T.C., Brettler, D.B., Sullivan, J.L., Desrosiers, R.C., 1995. Brief report: absence of intact nef sequences in a long-term survivor with nonprogressive HIV-1 infection. N. Engl. J. Med. 332 (4), 228-232.

Kosakovsky Pond, S.L., Frost, S.D., Muse, S.V., 2005. HyPhy: hypothesis testing using phylogenies. Bioinformatics 21 (5), 676-679.

Kuang, X.T., Li, X., Anmole, G., Mwimanzi, P., Shahid, A., Le, A.Q., Chong, L., Oian, H., Miura, T., Markle, T., Baraki, B., Connick, E., Daar, E.S., Jessen, H., Kelleher, A.D. Little, S., Markowitz, M., Pereyra, F., Rosenberg, E.S., Walker, B.D., Ueno, T. Brumme, Z.L., Brockman, M.A., 2014. Impaired Nef function is associated with early control of HIV-1 viremia. J. Virol. 88 (17), 10200-10213.

Leslie, A., Matthews, P.C., Listgarten, J., Carslon, J., Kadie, C., Ndung'u, T., Coovadia, H., Walker, B.D., Heckerman, D., Goulder, P.J.R., 2010. Additive contribution of HLA class I alleles in the immune control of HIV-1 infection. J. Virol. 84 9879-9888.

Lewis, M.J., Balamurugan, A., Ohno, A., Kilpatrick, S., Ng, H.L., Yang, O.O., 2008. Functional adaptation of Nef to the immune milieu of HIV-1 infection in vivo. J. Immunol. 180 (6), 4075-4081.

Lichterfeld, M., Yu, X.G., Cohen, D., Addo, M.M., Malenfant, J., Perkins, B., Pae, E. Johnston, M.N., Strick, D., Allen, T.M., Rosenberg, E.S., Korber, B., Walker, B.D., Altfeld, M., 2004. HIV-1 Nef is preferentially recognized by CD8 T cells in primary HIV-1 infection despite a relatively high degree of genetic diversity. AIDS 18 (10), 1383-1392.

Lundquist, C.A., Tobiume, M., Zhou, J., Unutmaz, D., Aiken, C., 2002. Nef-mediated downregulation of CD4 enhances human immunodeficiency virus type 1 replication in primary T lymphocytes. J. Virol. 76 (9), 4625-4633.

Mann, J.K., Byakwaga, H., Kuang, X.T., Le, A.Q., Brumme, C.J., Mwimanzi, P., Omarjee, S., Martin, E., Lee, G.O., Baraki, B., Danroth, R., McCloskey, R., Muzoora, C., Bangsberg, D.R., Hunt, P.W., Goulder, P.J., Walker, B.D., Harrigan, P.R. Martin, J.N., Ndung'u, T., Brockman, M.A., Brumme, Z.L., 2013. Ability of HIV-1 Nef to downregulate CD4 and HLA class I differs among viral subtypes. Retrovirology 10 (1), 100, http://dx.doi.org/10.1186/1742-4690-10-100.

Matthews, P.C., Prendergast, A., Leslie, A., Crawford, H., Payne, R., Rousseau, C. Rolland, M., Honeyborne, I., Carlson, J., Kadie, C., Brander, C., Bishop, K. Mlotshwa, N., Mullins, J.I., Coovadia, H., Ndung'u, T., Walker, B.D., Heckerman, D., Goulder, P.J.R., 2008. Central role of reverting mutations in HLA associations with human immunodeficiency virus set point. J. Virol. 82 (17), 8548-8559.

Mudd, P.A., Martins, M.A., Ericsen, A.J., Tully, D.C. Power, K.A., Bean, A.T. Piaskowski, S.M., Duan, L., Seese, A., Gladden, A.D., Weisgrau, K.L., Furlott, J.R. Kim, Y.I., Veloso de Santana, M.G., Rakasz, E., Capuano, S.r., Wilson, N.A. Bonaldo, M.C., Galler, R., Allison, D.B., Piatak, M.J., Haase, A.T., Lifson, J.D., Allen, T.M. Watkins, D.I., 2012. Vaccine-induced CD8 + T cells control AIDS virus replication. Nature 491 (7422), 129-133.

Mwimanzi, P., Hasan, Z., Hassan, R., Suzu, S., Takiguchi, M., Ueno, T., 2011a. Effects of naturally-arising HIV Nef mutations on cytotoxic T lymphocyte recognition and Nef's functionality in primary macrophages. Retrovirology 8, 50.

Mwimanzi, P., Hasan, Z., Hassan, R., Suzu, S., Takiguchi, M., Ueno, T., 2011b. Effects of naturally-arising HIV Nef mutations on cytotoxic T lymphocyte recognition and Nef's functionality in primary macrophages. Retrovirology 8, 50. http://dx.doi. org/10.1186/1742-4690-1188-1150.

Mwimanzi, P., Markle, T., Otsuka, H., Ogata, Y., Tokunaga, M., Miura, T., Martin, E., Pereyra, F., Walker, B., Brumme, Z., Brockman, M., Ueno, T., 2011c. Impairment of viral replication capacity by nef alleles from HIV elite controllers. Retrovirology 8 (Suppl2), P53. http://dx.doi.org/10.1186/1742-4690-1188-S1182P1153.

Mwimanzi, P., Markle, T.J., Martin, E., Ogata, Y., Kuang, X.T., Tokunaga, M., Mahiti, M., Pereyra, F., Miura, T., Walker, B.D., Brumme, Z.L., Brockman, M.A., Ueno, T. 2013. Attenuation of multiple Nef functions in HIV-1 elite controllers. Retrovirology 10 (1), 1.

Navis, M., Schellens, I.M., van Swieten, P., Borghans, J.A., Miedema, F., Kootstra, N.A., van Baarle, D., Schuitemaker, H., 2008. A nonprogressive clinical course in HIV infected individuals expressing human leukocyte antigen B57/5801 is associated with preserved CD8 + T lymphocyte responsiveness to the HW9 epitope in Nef. J. Infect. Dis. 197 (6), 871-879.

Noviello, C.M., Pond, S.L., Lewis, M.J., Richman, D.D., Pillai, S.K., Yang, O.O., Little, S.J., Smith, D.M., Guatelli, J.C., 2007. Maintenance of Nef-mediated modulation of major histocompatibility complex class I and CD4 after sexual transmission of human immunodeficiency virus type 1. J. Virol. 81 (9), 4776-4786.

Novitsky, V., Gilbert, P., Peter, T., McLane, M.F., Gaolekwe, S., Rybak, N., Thior, I., Ndung'u, T., Marlink, R., Lee, T.H., Essex, M., 2003. Association between virusspecific T-cell responses and plasma viral load in human immunodeficiency virus type 1 subtype C infection. J. Virol. 77 (2), 882-890.

Novitsky, V., Lagakos, S., Herzig, M., Bonney, C., Kebaabetswe, L., Rossenkhan, R., Nkwe, D., Margolin, L., Musonda, R., Moyo, S., Woldegabriel, E., van Widenfelt, E., Makhema, J., Essex, M., 2009a. Evolution of proviral gp120 over the first year of HIV-1 subtype C infection. Virology 383 (1), 47-59.

Novitsky, V., Wang, R., Margolin, L., Baca, J., Kebaabetswe, L., Rossenkhan, R., Bonney, C., Herzig, M., Nkwe, D., Moyo, S., Musonda, R., Woldegabriel, E., van Widenfelt, E., Makhema, J., Lagakos, S., Essex, M., 2009b. Timing constraints of in vivo gag mutations during primary HIV-1 subtype C infection. PLoS One 4 (11), e7727.

Novitsky, V., Woldegabriel, E., Kebaabetswe, L., Rossenkhan, R., Mlotshwa, B., Bonney, C., Finucane, M., Musonda, R., Moyo, S., Wester, C., van Widenfelt, E., Makhema, J., Lagakos, S., Essex, M., 2009c. Viral load and CD4+ T cell dynamics in primary HIV-1 subtype C infection. J. Acquir. Immune Defic. Syndr. 50, 65-76.

Pillay, T., Zhang, H., Drijfhout, J.W., Robinson, N., Brown, H., Khan, M., Moodley, J., Adhikari, M., Pfafferott, K., Feeney, M.E., St. John, A., Holmes, E.C., Coovadia, H. M., Klenerman, P., Goulder, P.J.R., Phillips, R.E., 2005. Unique acquisition of cytotoxic T-Lymphocyte escape mutants in infant human immunodeficiency virus type 1 infection. J. Virol. 79 (18), 12100-12105.

Radebe, M., Nair, K., Chonco, F., Bishop, K., Wright, J.K., van der Stok, M., Bassett, I.V., Mncube, Z., Altfeld, M., Walker, B.D., Ndung'u, T., 2011. Limited immunogenicity of HIV CD8 + T-cell epitopes in acute clade C virus infection. J. Infect. Dis. 204 (5), 768-776.

Sagar, M., 2010. HIV-1 transmission biology: selection and characteristics of infecting viruses. J. Infect. Dis. 202 (S2), S289-S296.

Sagar, M., Wu, X., Lee, S., Overbaugh, J., 2006. Human immunodeficiency virus type 1 V1-V2 envelope loop sequences expand and add glycosylation sites over the course of infection, and these modifications affect antibody neutralization sensitivity. J. Virol. 80 (19), 9586-9598.

Schneidewind, A., Brockman, M.A., Yang, R., Adam, I., Li, B., Le Gall, S., Rinaldo, C.R., Craggs, S.L., Allgaier, R.L., Power, K.A., Kuntzen, T., Tung, C.S., LaBute, M.X., Mueller, S.M., Harrer, T., McMichael, A.J., Goulder, P.J., Aiken, C., Brander, C., Kelleher, A.D., Allen, T.M., 2007. Escape from the dominant HLA-B27-restricted cytotoxic T-lymphocyte response in Gag is associated with a dramatic reduction in human immunodeficiency virus type 1 replication. J. Virol. 81, 12382-12393.

Storey, J.D., Tibshirani, R., 2003. Statistical significance for genomewide studies. Proc. Natl. Acad. Sci. USA 100, 9440-9445.

Swigut, T., Alexander, L., Morgan, J., Lifson, J., Mansfield, K.G., Lang, S., Johnson, R.P., Skowronski, J., Desrosiers, R., 2004. Impact of Nef-mediated downregulation of major histocompatibility complex class I on immune response to simian immunodeficiency virus. J. Virol. 78 (23), 13335-13344.

Treurnicht, F.K., Seoighe, C., Martin, D.P., Wood, N., Abrahams, M.-R., de Assis Rosa, D., Bredell, H., Woodman, Z., Hide, W., Mlisana, K., Abdool Karim, S., Gray, C.M., Williamson, C., 2010. Adaptive changes in HIV-1 subtype C proteins during early infection are driven by changes in HLA-associated immune pressure. Virology 396 (2), 213-225.

Ueno, T., Motozono, C., Dohki, S., Mwimanzi, P., Rauch, S., Fackler, O.T., Oka, S., Takiguchi, M., 2008. CTL-mediated selective pressure influences dynamic evolution and pathogenic functions of HIV-1 Nef. J. Immunol. 180, 1107-1116.

Veillette, M., Désormeaux, A., Medjahed, H., Gharsallah, N.E., Coutu, M., Baalwa, J., Guan, Y., Lewis, G., Ferrari, G., Hahn, B.H., Haynes, B.F., Robinson, J.E., Kaufmann, D.E., Bonsignori, M., Sodroski, J., Finzi, A., 2014. Interaction with cellular CD4 exposes HIV-1 envelope epitopes targeted by antibody-dependent cellmediated cytotoxicity. J. Virol. 88 (5), 2633-2644.

Watkins, R.L., Zou, W., Denton, P.W., Krisko, J.F., Foster, J.L., Garcia, J.V., 2013. In vivo analysis of highly conserved Nef activities in HIV-1 replication and pathogenesis. Retrovirology 10 (125), http://dx.doi.org/10.1186/1742-4690-1110-1125.

Wright, J.K., Brumme, Z.L., Carlson, J.M., Heckerman, D., Kadie, C.M., Brumme, C.J., Wang, B., Losina, E., Miura, T., Chonco, F., van der Stok, M., Mncube, Z., Bishop, K. Goulder, P.J.R., Walker, B.D., Brockman, M.A., Ndung'u, T., 2010. Gagprotease-mediated replication capacity in HIV-1 subtype C chronic infection: associations with HLA type and clinical parameters. J. Virol. 84 (20), 10820-10831.

Wright, J.K., Novitsky, V., Brockman, M.A., Brumme, Z.L., Brumme, C.J., Carlson, J.M., Heckerman, D., Wang, B., Losina, E., Leshwedi, M., van der Stok, M., Maphumulo, L., Mkhwanazi, N., Chonco, F., Goulder, P.J., Essex, M., Walker, B.D., Ndung'u, T., 2011. Influence of Gag-protease-mediated replication capacity on disease progression in individuals recently infected with HIV-1 subtype C. J. Virol 85, 3996-4006. 